\title{
The miR-1185-2-3p-GOLPH3L pathway promotes glucose metabolism in breast cancer by stabilizing p53-induced SERPINE1
}

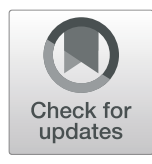

Youqin $\mathrm{Xu}^{1,2,3+}$, Wancheng Chen ${ }^{2,3+}$, Jing Liang ${ }^{4+}$, Xiaoqi Zeng ${ }^{5}$, Kaiyuan $\mathrm{Ji}^{6}$, Jianlong Zhou ${ }^{2,3}$, Shijun Liao ${ }^{5}$, Jiexian Wu', Kongyang Xing ${ }^{1}$, Zilong He ${ }^{7}$, Yang Yang ${ }^{2,3}$, Qianzhen Liư ${ }^{8}$, Pingyi Zhu' ${ }^{1}$, Yuchang Liu', Li Li', Minfeng Liu ${ }^{5^{*}}$, Wenxiao Chen ${ }^{1^{*}}$ and Wenhua Huang ${ }^{1,2,3,8^{*}}$ (1)

\begin{abstract}
Background: Phosphatidylinositol-4-phosphate-binding protein GOLPH3L is overexpressed in human ductal carcinoma of the breast, and its expression levels correlate with the prognosis of breast cancer patients. However, the roles of GOLPH3L in breast tumorigenesis remain unclear.

Methods: We assessed the expression and biological function of GOLPH3L in breast cancer by combining bioinformatic prediction, metabolomics analysis and RNA-seq to determine the GOLPH3L-related pathways involved in tumorigenesis. Dual-luciferase reporter assay and coimmunoprecipitation (Co-IP) were used to explore the expression regulation mechanism of GOLPH3L.

Results: We demonstrated that knockdown of GOLPH3L in human breast cancer cells significantly suppressed their proliferation, survival, and migration and suppressed tumor growth in vivo, while overexpression of GOLPH3L promoted aggressive tumorigenic activities. We found that miRNA-1185-2-3p, the expression of which is decreased in human breast cancers and is inversely correlated with the prognosis of breast cancer patients, is directly involved in suppressing the expression of GOLPH3L. Metabolomics microarray analysis and transcriptome sequencing analysis revealed that GOLPH3L promotes central carbon metabolism in breast cancer by stabilizing the p53 suppressor SERPINE1.
\end{abstract}

Conclusions: In summary, we discovered a miRNA-GOLPH3L-SERPINE1 pathway that plays important roles in the metabolism of breast cancer and provides new therapeutic targets for human breast cancer.

Keywords: Tumorigenesis, Glucose metabolism, Glycosylation, miRNA, p53-induced transcription

\footnotetext{
*Correspondence: matthewliu007@163.com; 602607747@qq.com;

huangwenhua2009@139.com

†Youqin $\mathrm{Xu}$, Wancheng Chen and Jing Liang contributed equally to this

work.

${ }^{5}$ Breast Center, Department of general surgery, Nanfang Hospital, Southern

Medical University, Guangzhou 510515, China

'Taishan People's Hospital, Postdoctoral Innovation Practice Base of Southern

Medical University, Taishan 529200, China

Full list of author information is available at the end of the article
}

(C) The Author(s). 2021 Open Access This article is licensed under a Creative Commons Attribution 4.0 International License, which permits use, sharing, adaptation, distribution and reproduction in any medium or format, as long as you give appropriate credit to the original author(s) and the source, provide a link to the Creative Commons licence, and indicate if changes were made. The images or other third party material in this article are included in the article's Creative Commons licence, unless indicated otherwise in a credit line to the material. If material is not included in the article's Creative Commons licence and your intended use is not permitted by statutory regulation or exceeds the permitted use, you will need to obtain permission directly from the copyright holder. To view a copy of this licence, visit http://creativecommons.org/licenses/by/4.0/ The Creative Commons Public Domain Dedication waiver (http://creativecommons.org/publicdomain/zero/1.0/) applies to the data made available in this article, unless otherwise stated in a credit line to the data. 


\section{Background}

Breast cancer is the most commonly diagnosed and most deadly cancer affecting women worldwide [1]. Tumor cells present a number of characteristics, such as selfproliferation ability [2], apoptosis resistance [3], unlimited replication potential [4], insensitivity to growth inhibition [5], continuous angiogenesis [6], tissue invasion [7] and metastasis [8]. Mammary tumorigenesis is a multistep process involving activation of oncogenes or inactivation of tumor suppressors, abnormal expression of noncoding RNA [9], loss of genome stability [10] and various genetic and epigenetic alterations [11]. Tumor cells prefer glycolysis for glucose metabolism under aerobic conditions (Warburg effect) rather than mitochondrial oxidative phosphorylation, which is more efficient for ATP production so that tumor cells can produce more energy and various metabolites in a short period of time [12]. ATP produced by glycolysis can satisfy high energy demand for rapid tumor growth. The intermediate products of glycolysis, such as glucose 6-phosphate and pyruvic acid, which can synthesize fatty acids and nucleic acids, regulate cell metabolism and biosynthesis. Moreover, glycolysis products acidify the microenvironment around the tumor, which is not conducive to the growth of normal cells but promotes tumor invasion and metastasis. The tumor suppressor protein p53 is the "guardian of the genome", which plays critical roles in cell cycle regulation, DNA repair, cell differentiation and apoptosis [13]. Tumor cells with p53 inactivation often show increasing glycolytic activity. Accumulating data show that p53 may confer tumor suppression by inhibiting the cancer metabolic switch from oxidative phosphorylation to glycolysis.

The Golgi apparatus is an important part of the cell membrane system involved in protein glycosylation, proteolytic activation and cellular secretory activity. Protein glycosylation is one of the most common posttranslational modifications, which regulates the location, function, activity, life span and diversity of proteins in tissues and cells and participates in various important life activities, such as cell recognition, differentiation, development, signal transduction and immune response. Glycosylation labels different proteins and changes the conformation of polypeptides to increase the stability of proteins. Mammalian proteins have three types of glycosylation: N-glycosylation (N-GlcNAc), O-glycosylation (O-GlcNAc) and glycosylphosphatidylinositol (GPI) anchor. In $\mathrm{N}$-glycosylation, the sugar chain is covalently linked to the free $\mathrm{NH}_{2}$ group of aspartic acid of the protein. The synthesis of the N-linked sugar chain starts from the endoplasmic reticulum (ER) and is completed at the Golgi. The glycoproteins formed by the ER have similar sugar chains. After entering the Golgi apparatus from the cis surface, most of the mannose on the original sugar chain is removed during the transport process between the membrane capsules. However, different types of sugar molecules are added to different glycosyltransferases to form oligosaccharide chains with different structures. $\mathrm{N}$-glycan biosynthesis coordinates the cellular response of tumor cells, determining growth, invasion and drug sensitivity [14]; for instance, Nacetyllactosamine $\mathrm{N}$-glycans mediate PD-L1 and PD-1, affecting the efficacy of anti-PD-L1 therapies [15]. Altering the $\mathrm{N}$-glycosylation of integrins affects cisinteractions with important membrane receptors, such as EGFR, contributing to tumor cell motility and migration [16]. The N-glycosylation product Fut8 is involved in the expression of cancer biomarkers as well as in the treatment of cancer, and GnT-V is highly associated with cancer metastasis, whereas GnT-III is associated with cancer suppression [17]. O-linked glycosylation takes place in the Golgi apparatus. The sugar chain is covalently linked to the free $\mathrm{OH}$ radical of serine/threonine residues in proteins. O-GlcNAc is responsible for cancer progression. O-GlcNAc modifications regulate the activities of FoxM1 and cyclin D1, which are involved in cell cycle progression and critical to cell proliferation [18]. O-GlcNAc has been implicated in cancer cell survival through the effect of hyper-O-GlcNAc via activation of $\mathrm{kB}$-mediated signaling [19]. Moreover, OGlcNAc participates in cancer cell invasion and metastasis by regulating E-cadherin trafficking and function [20]. Increased levels of O-GlcNAc transferase (OGT) have been found in breast cancer [19]. O-GlcNAcylation serves as a nutrient sensor to modulate crosstalk with phosphorylation, such as p53 [21]. The GPI glycosylphosphatidylinositol anchor is the only way for proteins to combine with the cell membrane, which is different from the general lipid modifying components, and its structure is extremely complex. Mutation in the X-linked PIGA gene increases the risk of developing leukemia [22]. The spatial structure of glycoprotein determines unique glycosyltransferases to initiate certain glycosylation modifications.

Current studies show that Golgi apparatus dysfunction is associated with tumor development, but more than $80 \%$ of Golgi-related proteins have not been reported to play a role in this process. Golgi phosphoprotein 3-like (GOLPH3L) is an important protein involved in the formation of Golgi vesicles and their anterograde transport to the plasma membrane. GOLPH3L is highly expressed in various tumor tissues and promotes the proliferation of rhabdomyosarcoma cells [23] and is involved in the regulation of proliferation, apoptosis and the cell cycle in cervical cancer cells [24]. Mechanistically, GOLPH3L has been reported as an activator of the NF- $\mathrm{B}$ signaling pathway in ovarian cancer [25]. Considering the critical roles of glycolysis in tumorigenesis, we demonstrate here 
that GOLPH3L contributes to tumorigenesis by promoting glucose metabolism in breast cancer by stabilizing certain downstream proteins of $\mathrm{p} 53$.

\section{Methods}

\section{BRC patient samples}

All patient-related studies were approved by the Institutional Review Board of Taishan People's Hospital and Nanfang Hospital of Southern Medical University.

\section{Human Cancer cell Xenograft model}

All animal work was approved by the Institutional Animal Care and Use Committee (IACUC) of Southern Medical University. A total of $5 \times 10^{6}$ breast cancer cells were implanted into the skeletal muscle of the hind limbs of 3 4-week-old BALB/c nude mice (nu/nu). One week after transplantation, the diameter of tumors was measured every 3 days. Tumors were recovered and weighed after 3 weeks.

\section{Cell culture}

Human normal mammary epithelial cell line (MCF-10A) and human breast cancer cell lines (T47D, BT474, MCF7, MDA-MB-231, SK-BR-3) were purchased from American Type Culture Collection (ATCC, Manassas, VA, USA). Human breast cancer cell lines (T47D, BT474, MCF-7, MDA-MB-231, SK-BR-3) were cultured in Roswell Park Memorial Institute 1640 medium (Gibco, USA) supplemented with 10\% fetal bovine serum (FBS, HyClone, Utah, USA) and $1 \%$ penicillinstreptomycin (Pen/Strep) (Gibco) at $37^{\circ} \mathrm{C}$ with $5 \% \mathrm{CO}_{2}$. The human normal mammary epithelial cell line MCF10A was cultured in media and supplements from the MEGM kit (Lonza/Clonetics, CC-3150) and 10\% FBS supplemented with $100 \mathrm{ng} / \mathrm{ml}$ cholera toxin (Sigma-Aldrich, C8052) at $37^{\circ} \mathrm{C}$ with $5 \% \mathrm{CO}_{2}$.

\section{Establishment of transfected cell lines}

The vectors expressing GOLPH3L-specific siRNA (RIBOBIO, Cat\# siG000055204A-C) and SERPINE1-specific siRNA (ThermoFisher, Cat\# 4390771). Vectors expressing human GOLPH3L cDNA and SERPINE1 cDNA were transfected into cells as previously described [26]. Cells were selected with puromycin $(2 \mu \mathrm{g} / \mathrm{ml}$, GeneChem) for 3 days for stable transfection.

\section{Western blot analysis}

Cells were extracted for total protein analysis using lysis buffer, and samples were separated on $8-15 \%$ SDSPAGE and transferred to nitrocellulose membranes, which were blocked with blocking buffer (5\% skim milk in PBS with $0.05 \%$ Tween 20) and incubated with primary antibody in the blocking buffer. After being washed three times with blocking buffer, the membrane was probed with secondary antibody and developed with Supersignal West Pico or Dura (Thermo Fisher Scientific).

\section{Quantitative PCR analysis}

Real-time PCR analysis was performed using the StepOnePlus Real-Time PCR System (Applied Biosystems) with FastStart Universal SYBR Green Master Mix (Roche) as previously reported. The primer sets used were as follows:

GAPDH-F: GAACGGGAAGCTCACTGG; GAPDH-R: GCCTGCTTCACCACCTTCT; GOLPH3L-F: GTAAATGACCCTCAGCGTATGG; GOLPH3L-R: GTTCTACTAAGTCCTTGGCTCGAT; miRNA-1185-2-3p: AUAUACAGGGGGAGACUCUCAU; miRNA-1185-2-3p-F: ACACTCCAGCTGGGATAT ACAGGGGGAGAC; miRNA-1185-2-3p-R: CTCAACTGGTGTCGTGGA; U6-F: GCTTCGGCAGCACATATACTAAAAT;

U6-R: CGCTTCATGAATTTGCGTGTCAT; miRNA-1185-2-3p mimic-F: AUAUACAGGGGGAG ACUCUCAU; miRNA-1185-2-3p mimic-R: GAGAGUCUCCCCCU GUAUAUUU; miRNA-1185-2-3p inhibitor: AUGAGAGUCUCCCC CUGUAUAU. SERPINE1-F: ACCGCAACGTGGTTTTCTCA; SERPINE1-R: TTGAATCCCATAGCTGCTTGAAT.

The PCR conditions were as follows: $10 \mathrm{~min}$ at $95^{\circ} \mathrm{C}$, 40 cycles of $15 \mathrm{~s}$ at $95^{\circ} \mathrm{C}$, and $1 \mathrm{~min}$ at $60^{\circ} \mathrm{C}$. The average $\mathrm{Ct}$ value for each gene was determined from triplicate reactions and normalized to that of $\beta$-actin for genes and U6 for microRNAs (miRNAs).

\section{Cell proliferation, apoptosis, migration assay and cell cycle assay}

For EdU-high content screening of the cellular proliferation assay, cells were prepared by trypsinization and seeded onto 96-well plates at a density of $1 \times 10^{4}$ cells per well. After incubation for $48 \mathrm{~h}$ at $37^{\circ} \mathrm{C}$, the old medium was discarded, and $100 \mu \mathrm{l}$ of medium containing EdU was added to each well and incubated for $6 \mathrm{~h}$. Cells were fixed with paraformaldehyde at room temperature for $20 \mathrm{~min}$. Then, $100 \mu \mathrm{l}$ of $2 \mathrm{mg} / \mathrm{ml}$ glycine solution and $100 \mu \mathrm{l}$ of $0.5 \%$ Triton X-100 solution were added to the wells separately, and the cells were washed twice with PBS. One hundred microliters of Apollo-643 staining solution was added in each well for $30 \mathrm{~min}$ and then discarded. After destaining and rinsing, DAPI reaction mixture was added to each well and incubated for 30 min. A GE IN CELL Analyzer 6500HS Confocal High 
Content Imaging Analyzer was used for collecting images.

Consistent treatments were applied to prepared cells in 96-well plates: $100 \mu \mathrm{l} 2 \mathrm{mg} / \mathrm{ml}$ glycine solution and $100 \mu \mathrm{l} 0.5 \%$ Triton X-100 solution were added into wells separately, and then the cells were washed twice with PBS. Fluorescein-dUTP solution $(50 \mu \mathrm{l})$ mixed with TdT enzyme was added to each well and incubated for $2 \mathrm{~h}$ at room temperature. Then, the reaction solution was discarded, and $100 \mu \mathrm{l} 2 \times$ SSC buffer was immediately added into each well. A $1 \times$ DAPI reaction mixture was added to the plates and incubated for $30 \mathrm{~min}$. A GE IN CELL Analyzer 6500HS Confocal High Content Imaging Analyzer was used for collecting images after the cells were washed three times with PBS.

Consistent treatments were applied to prepared cells in 24-well plates at a density of $2.5 \times 10^{4}$ cells per well. After adjusting the cell concentration to $1 \times 10^{5}$ cells $/ \mathrm{ml}$, the cells were seeded into the upper chamber at $1 \times 10^{4}$ cells/ $\mu \mathrm{l}$, and the lower chamber was immediately filled with $150 \mu \mathrm{l}$ complete medium with $10 \%$ FBS as a chemoattractant and then incubated for $48 \mathrm{~h}$. After the medium was discarded from the lower chamber, $150 \mu \mathrm{l}$ PBS was added to each well for $5 \mathrm{~min}$. Calcein AM cell staining solution at a working concentration of $2.5 \mu \mathrm{M}$ was prepared with complete medium, and $150 \mu \mathrm{l}$ staining solution was added to each well for incubation at room temperature for $30 \mathrm{~min}$ after the PBS was discarded. After incubation, $150 \mu$ l trypsin was added to the lower chamber and incubated at $37^{\circ} \mathrm{C}$ for $15 \mathrm{~min}$. Serum-containing medium was added to the chamber to stop digestion. Cells in the subchamber were aspirated for cell counting.

For the cell cycle assay, cells were prepared in 96-well plates at a density of $1 \times 10^{4}$ cells per well. After being washed with PBS one time, the cells were fixed with $4 \%$ paraformaldehyde for $20 \mathrm{~min}$; the paraformaldehyde was removed, and $100 \mu \mathrm{l}$ of $2 \mathrm{mg} / \mathrm{ml}$ glycine solution was added. The glycine solution was subsequently removed, and the cells were washed with PBS one time. Then, $100 \mu \mathrm{l}$ of $0.5 \%$ Triton X-100 PBS solution was added to each well, which were then washed twice with PBS. One hundred microliters of $1 \times$ DAPI reaction mixture was added to each well, and the plates were incubated for 30 min. After being washed with PBS three times, a GE IN CELL Analyzer 6500HS Confocal High Content Imaging Analyzer was used to collect the images.

\section{Dual-luciferase reporter assay}

Cells were seeded in triplicate onto 6 -well plates at a density of $4 \times 10^{5}$ cells/well for $48 \mathrm{~h}$ and transfected with $0.3 \mu \mathrm{g}$ of REPOTM-AP-1-luc plasmid and controlluciferase plasmid separately together with $30 \mathrm{ng}$ of pGMR TK renilla plasmid (GenomeDitech, Shanghai,
China) using Lipofectamine 3000 reagent (Invitrogen, Carlsbad, USA). Firefly and Renilla luciferase activities were measured using the Dual-Luciferase Reporter Assay Kit (Promega, Madison, USA) after $48 \mathrm{~h}$ of transfection.

\section{Metabolomic analysis}

Cells were collected in centrifuge tubes filled with $1 \mathrm{ml}$ methanol:acetonitrile:water $(2: 2: 1, \mathrm{~V} / \mathrm{V})$ at a density of $2 \times 10^{7}$ cells/tube after washing with PBS and physiological saline solution. The liquid nitrogen is stored at $80^{\circ} \mathrm{C}$ after quick freezing. To analyze metabolomics, samples were analyzed at Applied Protein Technology (APT, Shanghai). Partial least squares discrimination analysis was applied to reveal the relationship between the expression of metabolites and sample types by calculating the cast variable importance for the projection (VIP) to measure the expression pattern of each metabolite for each group. All differentially expressed metabolites were selected for Gene Ontology (GO) and Kyoto Encyclopedia of Genes and Genomes (KEGG) pathway analyses (VIP score $>1.0$ ). GO was performed with KOBAS3.0 software. The differentially expressed metabolites and enriched pathways were mapped using the KEGG pathways with KOBAS3.0 software (http://www. genome.jp/kegg).

\section{Transcriptome sequencing}

Total RNA was isolated from cells/tissues using TRIzol (Invitrogen) according to the manufacturer's protocol. RNA purity was assessed using the ND-1000 Nanodrop. Each RNA sample had an A260:A280 ratio above 1.8 and an A260:A230 ratio above 2.0. RNA integrity was evaluated using the Agilent 2200 TapeStation (Agilent Technologies, USA), and each sample had an RNA integrity number (RIN) above 7.0. Briefly, rRNAs were removed from total RNA using the EpicentreRibo-Zero rRNA Removal Kit (Illumina, USA) and fragmented to approximately $200 \mathrm{bp}$. Subsequently, the purified RNAs were subjected to first strand and second strand cDNA synthesis followed by adaptor ligation and enrichment with a low-cycle according to the instructions of the NEBNext $^{\circ}$ Ultra $^{\text {Tux }}$ RNA Library Prep Kit for Illumina (NEB, USA). The purified 1 library products were evaluated using the Agilent 2200 TapeStation and Qubit ${ }^{\circ} 2.0$ (Life Technologies, USA) and then diluted to $10 \mathrm{pM}$ for cluster generation in situ on the pair-end flow cell followed by sequencing $(2 \times 150 \mathrm{bp})$ with a HiSeq3000. Clean reads were obtained after removal of reads containing adaptor, poly- $\mathrm{N}$ and low quality from raw data. HISAT2 was used to align the clean reads to the mouse reference genome $\mathrm{mm} 10$ with default parameters. HTSeq was subsequently employed to convert aligned short reads into read counts for each gene model. Differential expression was assessed by DEseq using read 
counts as input. The Benjamini-Hochberg multiple test correction method was enabled. Differentially expressed genes were chosen according to the criteria of fold change $>2$ and adjusted $p$-value $<0.05$. All the differentially expressed genes were used for heat map analysis and KEGG ontology enrichment analyses. For KEGG enrichment analysis, a $p$-value $<0.05$ was used as the threshold to determine significant enrichment of the gene sets.

\section{Seahorse assay}

To measure the levels of glycolytic ATP production, $1 \times$ $10^{4}$ cells were seeded into each well of black 96-well plates. To normalize the levels of protein, the same number of cells were seeded into clear bottom 96-well plates. Cells were incubated in medium containing $1 \mu \mathrm{M}$ oligomycin (Sigma-Aldrich) to inhibit mitochondrial oxidative ATP production or $25 \mathrm{mM}$ 2-deoxy-D-glucose (2DG) to inhibit glycolytic ATP production. After washing the cells with PBS, ATP levels were measured using a kit according to the manufacturer's protocol (PerkinElmer). Total ATP production was calculated by subtracting the amount of ATP in cells treated with both oligomycin and 2-DG from the amount of ATP in cells without treatment. To normalize the number of cells, the protein concentration was measured using the Bicinchoninic Acid Protein Assay Kit (BCA, Sigma-Aldrich) according to the manufacturer's protocols.

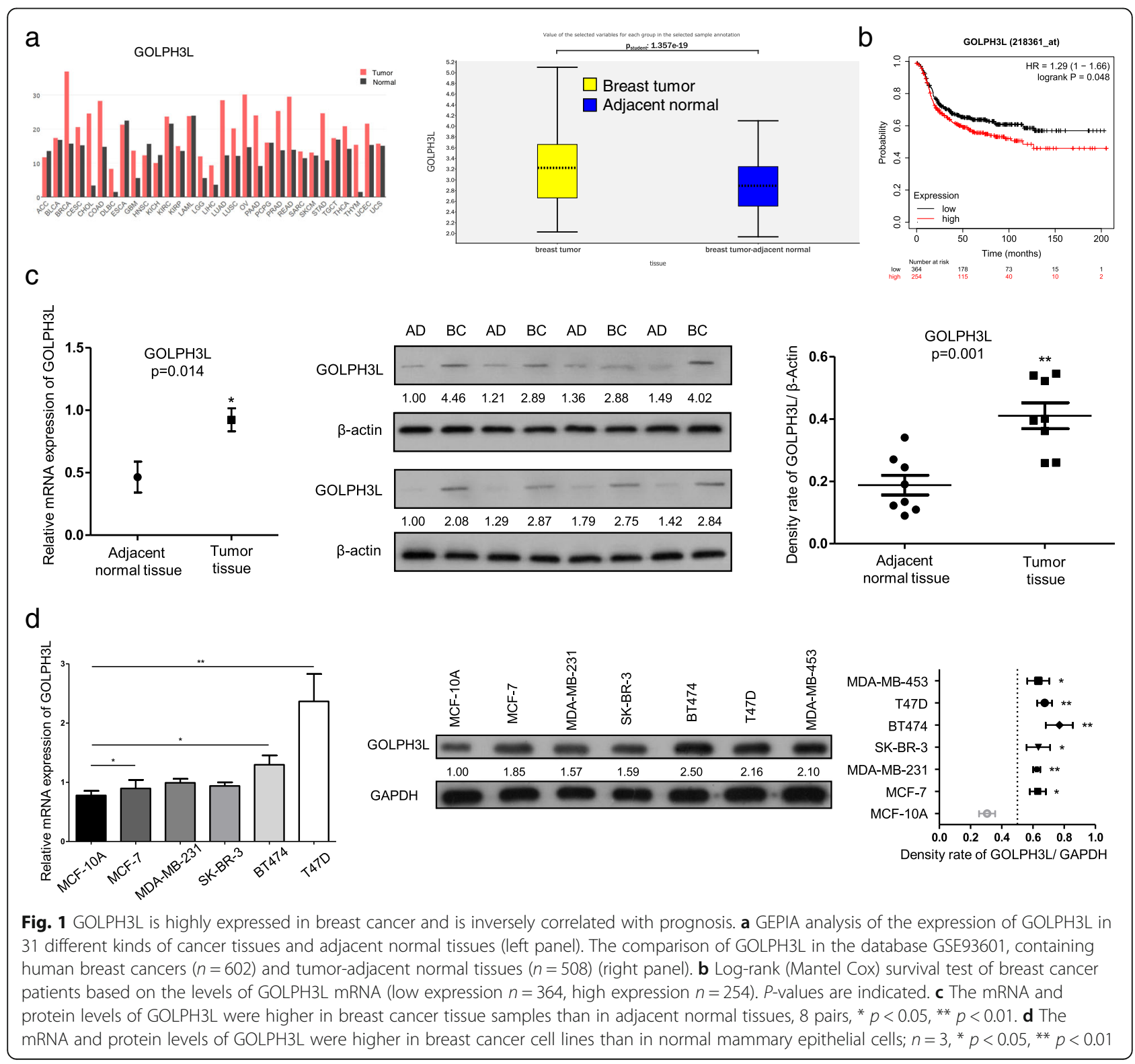




\section{Protein stability analysis}

Cells were transfected with siRNAs, and cyclohexamide (CHX; 1:1000) was used to treat cells, which were harvested at various time points $(0 \mathrm{~h}, 0.5 \mathrm{~h}, 1 \mathrm{~h}, 2 \mathrm{~h}, 4 \mathrm{~h}, 6 \mathrm{~h}$ and $12 \mathrm{~h}$ ). Levels of various proteins were determined by Western blot analysis and quantified with ImageJ software.

\section{Coimmunoprecipitation assay}

Immunoprecipitation assays were performed as previously described [27]. Briefly, cells were lysed in RIPA duffer containing protease and phosphatase inhibitors, and cells were collected after centrifugation at $12,000 \times \mathrm{g}$ for $10 \mathrm{~min}$ at $4{ }^{\circ} \mathrm{C}$. Supernatants were immunoprecipitated with antibodies followed by incubation with magnetic protein $\mathrm{A} / \mathrm{G}$ beads (Pierce) for $2 \mathrm{~h}$ at $4{ }^{\circ} \mathrm{C}$. The immune complexes were washed three times with PBS, resuspended in SDS-PAGE buffer and assessed by Western blot analysis.

\section{Statistical analysis}

Data were analyzed using SPSS 20.0. and two-tailed independent Student's t-test; $P<0.05$ was considered significant. Two patient cohorts were compared by KaplanMeier survival plot, and log-rank $p$-values were calculated.

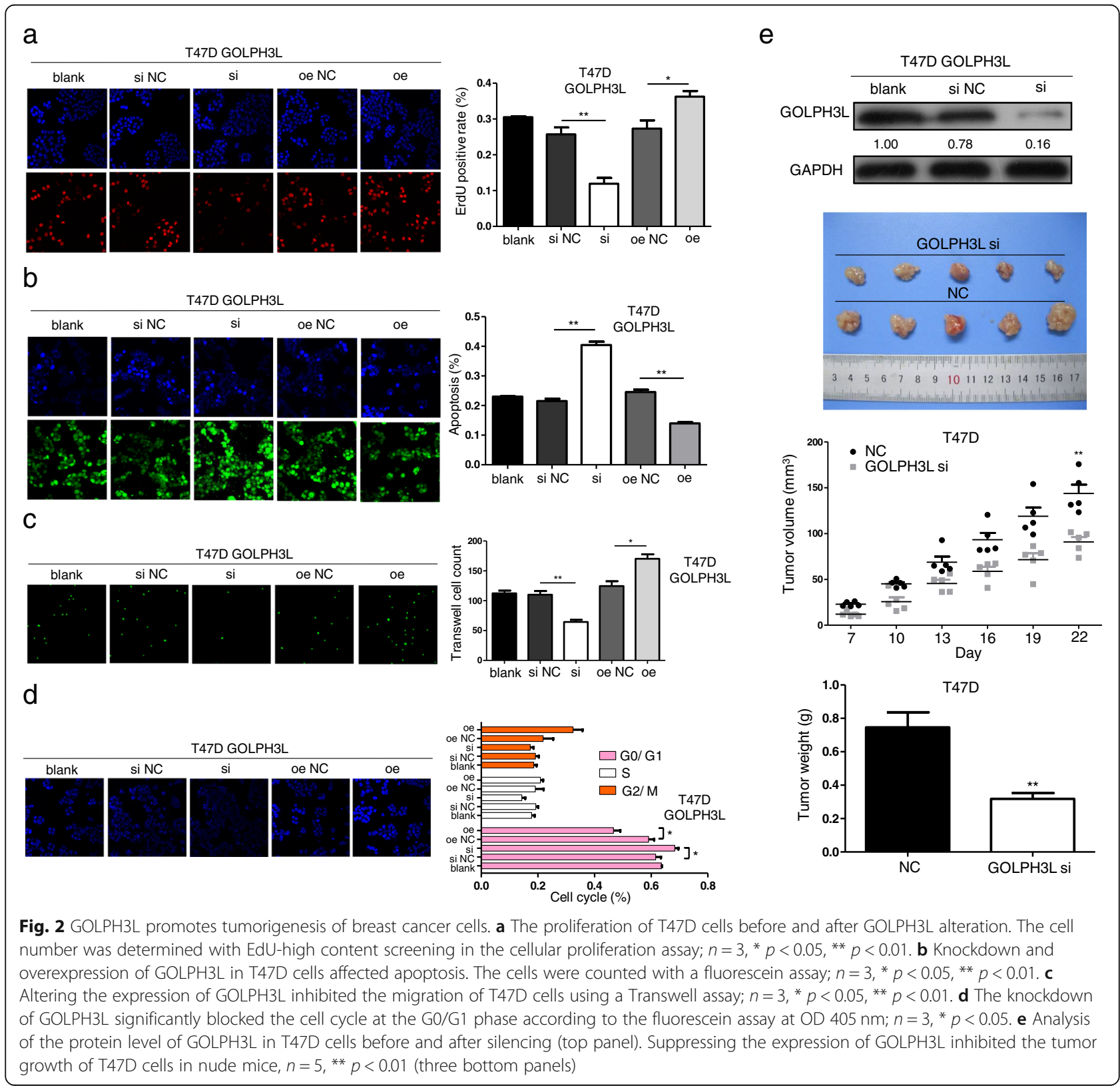




\section{Results}

GOLPH3L is highly expressed in breast Cancer and promotes the tumorigenesis of breast Cancer cells Using Gene Expression Profiling Interactive Analysis (GEPIA, http://gepia.cancer-pku.cn/index.html) and profiling the expression data of GOLPH3L in breast tumors and tumor-adjacent normal tissues in the database (GSE93601), we confirmed that GOLPH3L was dramatically overexpressed in breast cancer samples compared to normal control samples (Fig. 1a). Moreover, the expression levels of GOLPH3L were inversely correlated with the prognosis of human breast cancer patients (Fig. 1b). In this context, GOLPH3L promotes breast tumorigenesis. To explore the explicit roles of GOLPH3L in breast tumorigenesis, we investigated the
mRNA and protein expression levels of GOLPH3L in breast cancer tissues and paired adjacent normal tissues as well as in various breast cancer cell lines and normal breast epithelial cell lines. The relative mRNA and protein expression of GOLPH3L in cancer tissues was much higher than that in adjacent normal tissues (Fig. 1c). Consistently, compared to the normal breast epithelial cell line MCF-10A, the expression levels of GOLPH3L mRNA and protein were increased in breast cancer cell lines, especially in T47D and BT474 cells (Fig. 1d).

Therefore, we examined the roles of GOLPH3L in T47D and BT474 cells by altering the expression of GOLPH3L through knockdown or overexpression (Figure $\mathrm{S} 1 \mathrm{a}$ and $\mathrm{b}$ ). Restraining GOLPH3L expression in breast cells decreased cellular proliferation and survival,

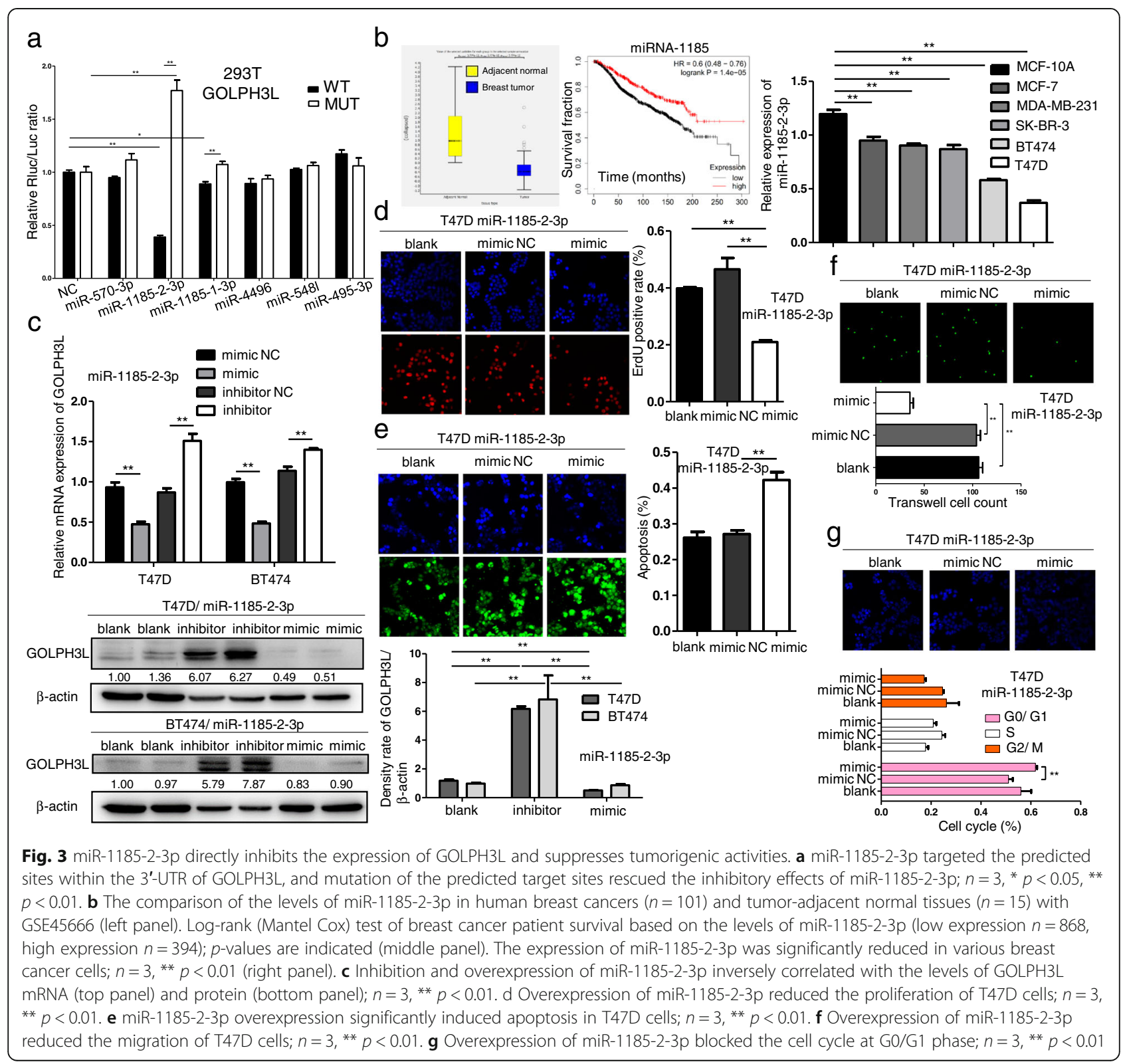


while upregulating the expression of GOLPH3L in T47D cells promoted proliferation and survival (Fig. 2a and b). Transwell assays revealed that silencing GOLPH3L inhibited the migration of T47D cells, and in contrast, overexpressing GOLPH3L in cells obviously facilitated cancer cell migration (Fig. 2c). Moreover, knockdown of GOLPH3L expression significantly blocked the cell cycle at the G0/G1 phase (Fig. 2d). Further evidence of the role of GOLPH3L in tumorigenesis is that knockdown of GOLPH3L in T47D cells significantly reduced the growth of tumors formed by T47D cells in immunodeficient mice (Fig. 2e). Consistent data were obtained using BT474 breast cancer cells (Figure S1c-f). These findings demonstrate that GOLPH3L promotes the tumorigenesis of breast cancer cells in various ways.
miRNA-1185-2-3p negatively regulates GOLPH3L and positively correlates with the prognosis of human breast Cancer patients

miRNAs are 19-25 nucleotide-long noncoding RNAs that regulate gene expression via messenger RNA degradation and translation. miRNAs are involved in every aspect of biological processes, and it is worth noting that alterations in miRNA expression induce the initiation, progression and metastasis of human tumors. Considering the consequence of GOLPH3L in promoting breast tumorigenesis, we utilized the miRDB prediction program (http://mirdb.org/). Based on the predicted results (Figure S2a), we mutated the target sites of the top five predicted miRNAs in the 3'-UTR of GOLPH3L mRNA. A dual-luciferase

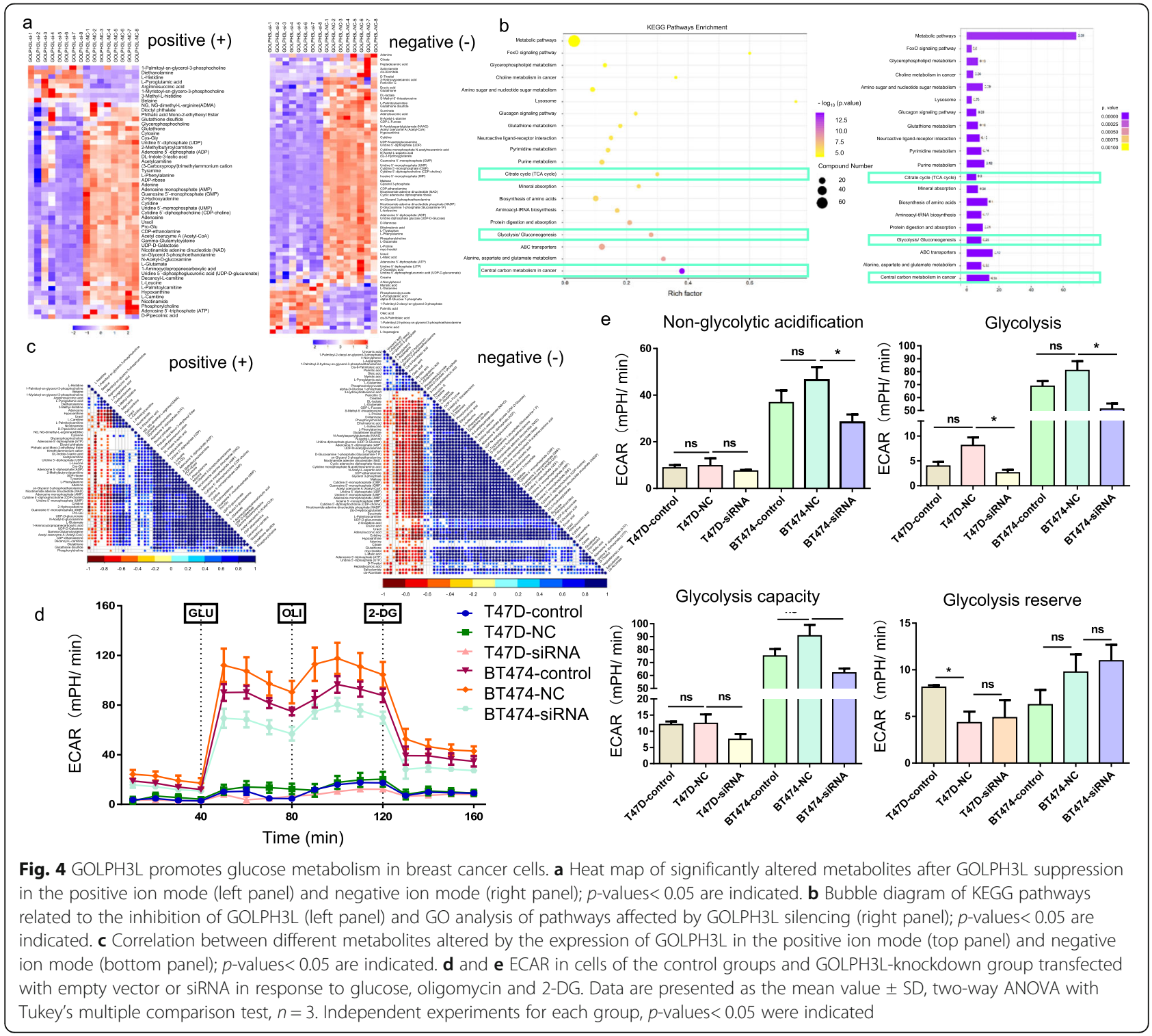


Table 1 Metabolites that were altered significantly after GOLPH3L silencing

\begin{tabular}{|c|c|c|c|c|}
\hline Name & ID & VIP & Fold change & $p$-value \\
\hline M348T450 & Adenosine monophosphate (AMP) & 5.35017309 & 0.343953619 & $1.85022 \mathrm{E}-09$ \\
\hline M489T438 & Cytidine 5'-diphosphocholine (CDP-choline) & 3.769023161 & 0.294683065 & 2.09147E-09 \\
\hline M325T436 & Uridine 5'-monophosphate (UMP) & 2.859910755 & 0.36416908 & $1.85344 \mathrm{E}-08$ \\
\hline M136T425 & Adenine & 3.6245555449 & 0.541578913 & 3.52988E-08 \\
\hline M216T387 & sn-Glycerol 3-phosphoethanolamine & 3.817810077 & 0.662660731 & $1.64313 \mathrm{E}-07$ \\
\hline M113T160 & Uracil & 2.339930939 & 0.254999039 & 2.66289E-07 \\
\hline M162T355_2 & L-carnitine & 2.259917766 & 0.687860774 & $5.55508 \mathrm{E}-07$ \\
\hline M244T235 & Cytidine & 1.320380286 & 0.343617832 & $6.16343 \mathrm{E}-07$ \\
\hline M664T430 & Nicotinamide adenine dinucleotide (NAD) & 5.426903613 & 0.531267518 & $6.8003 \mathrm{E}-07$ \\
\hline M204T426 & $\mathrm{N}$-acetyl-D-glucosamine & 2.162566187 & 0.585855735 & $6.80877 \mathrm{E}-07$ \\
\hline M268T167 & Adenosine & 2.080090077 & 0.506337679 & $8.19558 \mathrm{E}-07$ \\
\hline M152T457 & 2-Hydroxyadenine & 1.192073219 & 0.418366736 & $1.00101 \mathrm{E}-06$ \\
\hline M364T457 & Guanosine 5'-monophosphate (GMP) & 2.20531908 & 0.381298814 & $1.34425 \mathrm{E}-06$ \\
\hline M598T474 & Uridine 5'-diphosphoglucuronic acid (UDP-D-glucuronate) & 1.241389474 & 0.627904863 & 2.896E-06 \\
\hline M810T418 & Acetyl coenzyme A (Acetyl-CoA) & 1.148074386 & 0.537400888 & 7.08625E-06 \\
\hline M148T391 & L-glutamate & 3.853584751 & 0.737242788 & $8.94063 \mathrm{E}-06$ \\
\hline M584T438 & UDP-D-galactose & 1.033843982 & 0.641425356 & $9.98778 \mathrm{E}-06$ \\
\hline M137T166 & Hypoxanthine & 6.446185931 & 0.11746834 & $1.93784 \mathrm{E}-05$ \\
\hline M428T452_2 & Adenosine 5'-diphosphate (ADP) & 7.163025043 & 0.57751916 & $2.09404 \mathrm{E}-05$ \\
\hline M447T442 & CDP-ethanolamine & 1.026217107 & 0.611693587 & $2.16949 \mathrm{E}-05$ \\
\hline M291T464 & Argininosuccinic acid & 1.026399168 & 1.525518377 & $2.31077 \mathrm{E}-05$ \\
\hline M188T257 & DL-indole-3-lactic acid & 1.240956561 & 0.489217809 & $2.34219 \mathrm{E}-05$ \\
\hline M102T391 & 1-Aminocyclopropanecarboxylic acid & 1.410081315 & 0.756256635 & $2.38876 \mathrm{E}-05$ \\
\hline M246T242 & 2-Methylbutyroylcarnitine & 7.369334193 & 0.509326233 & $3.52739 \mathrm{E}-05$ \\
\hline M106T299 & Diethanolamine & 1.53304285 & 14.44114449 & $3.92078 \mathrm{E}-05$ \\
\hline M245T409 & Pro-Glu & 1.083816026 & 0.642279716 & $4.4156 \mathrm{E}-05$ \\
\hline M316T193 & Decanoyl-L-carnitine & 1.705457563 & 0.620089802 & 8.50963E-05 \\
\hline M204T303 & Acetylcarnitine & 10.77036712 & 0.725339373 & 0.000102368 \\
\hline M422T466 & Uridine 5'-diphosphate (UDP) & 1.109528766 & 0.668781099 & 0.000142935 \\
\hline M400T159_2 & L-palmitoylcarnitine & 8.34588634 & 0.644174553 & 0.000177908 \\
\hline M147T369 & L-pyroglutamic acid & 1.765274591 & 1.542951135 & 0.000179981 \\
\hline M233T395 & Gamma-glutamylcysteine & 1.388401872 & 0.540837694 & 0.000320962 \\
\hline M112T236 & Cytosine & 1.593691839 & 0.555964705 & 0.000415561 \\
\hline M542T430 & ADP-ribose & 1.028412857 & 0.622601002 & 0.00064559 \\
\hline M308T428 & Glutathione & 4.266509616 & 0.504989177 & 0.000716522 \\
\hline M123T61 & Nicotinamide & 1.851347574 & 0.566997397 & 0.000943254 \\
\hline M156T441 & L-histidine & 1.2602915 & 1.504208252 & 0.00100569 \\
\hline М170T397 & 3-Methyl-L-histidine & 2.097791259 & 7.382253419 & 0.001046756 \\
\hline M120T256 & Tyramine & 1.693843786 & 0.657319643 & 0.001442055 \\
\hline M166T256 & L-phenylalanine & 1.120111864 & 0.675428936 & 0.002216965 \\
\hline M146T374_2 & (3-Carboxypropyl) trimethylammonium cation & 3.995126207 & 0.750604189 & 0.002386475 \\
\hline M132T263 & L-leucine & 1.432367955 & 0.424002729 & 0.002807843 \\
\hline M179T427 & Cys-Gly & 1.127437605 & 0.643923687 & 0.006537627 \\
\hline M258T457 & Glycerophosphocholine & 1.162325024 & 0.70923693 & 0.018142168 \\
\hline
\end{tabular}


Table 1 Metabolites that were altered significantly after GOLPH3L silencing (Continued)

\begin{tabular}{|c|c|c|c|c|}
\hline Name & ID & VIP & Fold change & $p$-value \\
\hline M508T581 & Adenosine 5'-triphosphate (ATP) & 1.252149198 & 0.44136226 & 0.018216171 \\
\hline M391T33 & Dioctyl phthalate & 5.057833924 & 0.806525311 & 0.019249997 \\
\hline M613Т491 & Glutathione disulfide & 2.467281297 & 0.722876989 & 0.059115948 \\
\hline M468T200 & 1-Myristoyl-sn-glycero-3-phosphocholine & 1.668722344 & 2.054758707 & 0.059687763 \\
\hline M118T462 & Betaine & 2.078707794 & 1.308639016 & 0.05975974 \\
\hline M184T566 & Phosphorylcholine & 1.969089209 & 0.217723196 & 0.060512883 \\
\hline M496T166 & 1-Palmitoyl-sn-glycero-3-phosphocholine & 3.467532932 & 1.405433036 & 0.060816012 \\
\hline M203T505 & NG,NG-dimethyl-L-arginine (ADMA) & 1.02231111 & 0.612169445 & 0.079805836 \\
\hline M130T542 & D-pipecolinic acid & 1.22345702 & 0.462637578 & 0.081203784 \\
\hline M279T33 & Phthalic acid mono-2-ethylhexyl ester & 2.038184914 & 0.869293803 & 0.088733314 \\
\hline M341T395 & maltose & 2.111953392 & 0.028561128 & 3.10543E-17 \\
\hline M347T439 & Inosine 5'-monophosphate (IMP) & 3.625592991 & 0.301881382 & 6.71817E-16 \\
\hline M322T450 & Cytidine 5'-monophosphate (CMP) & 1.192275542 & 0.62674391 & $2.23826 \mathrm{E}-14$ \\
\hline M662T429 & Nicotinamide adenine dinucleotide (NAD) & 5.135975169 & 0.567384075 & 4.63725E-13 \\
\hline M547T437 & Cytidine 5'-diphosphocholine (CDP-choline) & 1.791062321 & 0.406528182 & 1.40724E-12 \\
\hline M362T455 & Guanosine 5'-monophosphate (GMP) & 3.653583959 & 0.407242856 & 4.41861E-12 \\
\hline M540T428 & Cyclic adenosine diphosphate ribose & 4.068411598 & 0.566724985 & $6.8843 \mathrm{E}-12$ \\
\hline M606T424 & UDP-N-acetylglucosamine & 18.47592875 & 0.611830043 & 7.85683E-12 \\
\hline M323T434 & Uridine 5'-monophosphate (UMP) & 7.171175055 & 0.404982084 & $1.33639 \mathrm{E}-11$ \\
\hline M346T422 & Adenosine monophosphate (AMP) & 14.34548448 & 0.318588959 & 2.04199E-11 \\
\hline M203T254 & L-tryptophan & 2.120508327 & 0.500782842 & 3.29102E-11 \\
\hline M214T388 & sn-Glycerol 3-phosphoethanolamine & 9.759185833 & 0.725105379 & $3.90018 \mathrm{E}-11$ \\
\hline M242T234 & Cytidine & 1.768227149 & 0.351697024 & 1.48152E-10 \\
\hline M445T440 & CDP-ethanolamine & 2.832548838 & 0.60943293 & $2.32647 \mathrm{E}-10$ \\
\hline M808T416 & Acetyl coenzyme A (acetyl-CoA) & 1.321582442 & 0.559526257 & 3.85093E-10 \\
\hline M742T485 & Nicotinamide adenine dinucleotide phosphate (NADP) & 1.711544078 & 0.624512533 & 8.56497E-10 \\
\hline M130T260 & L-Isoleucine & 3.729655739 & 0.588743745 & 1.07604E-09 \\
\hline M613T434 & Cytidine monophosphate $\mathrm{N}$-acetylneuraminic acid & 3.365736279 & 0.515824367 & $1.14679 \mathrm{E}-09$ \\
\hline M171Т383 & Glycerol 3-phosphate & 3.123463887 & 0.690487464 & 1.17292E-09 \\
\hline M147T385 & (S)-2-Hydroxyglutarate & 2.209506147 & 0.672217978 & $1.29056 \mathrm{E}-09$ \\
\hline M167T451 & Phosphoenolpyruvate & 3.418641375 & 1.879691109 & 1.38673E-09 \\
\hline M174T387_2 & N-Acetyl-L-aspartic acid & 5.248401418 & 0.659295634 & 2.85219E-09 \\
\hline M258T410 & D-Glucosamine 1-phosphate (glucosamine-1P) & 1.215448728 & 0.595730278 & 2.98007E-09 \\
\hline M135T165 & Hypoxanthine & 2.83515564 & 0.155797031 & $3.3521 \mathrm{E}-09$ \\
\hline M565T436 & Uridine diphosphate glucose (UDP-D-glucose) & 4.94082239 & 0.593931258 & $5.65669 \mathrm{E}-09$ \\
\hline M239T387 & D-Mannose & 1.267493145 & 0.837341404 & 1.65544E-08 \\
\hline M131T253 & Ethylmalonic acid & 1.942789906 & 0.670553701 & $2.25702 \mathrm{E}-08$ \\
\hline M182T474 & Phosphorylcholine & 1.592146407 & 0.776441511 & $5.19225 \mathrm{E}-08$ \\
\hline M426T481 & Adenosine 5'-diphosphate (ADP) & 2.142482016 & 0.786728943 & $6.27755 \mathrm{E}-08$ \\
\hline M403T463 & Uridine 5'-diphosphate (UDP) & 6.023335277 & 0.590303313 & $6.92096 \mathrm{E}-08$ \\
\hline M164T253 & L-phenylalanine & 2.745272587 & 0.649759377 & 1.09694E-07 \\
\hline M128T370_1 & L-pyroglutamic acid & 1.084478181 & 1.326574096 & $1.35422 \mathrm{E}-07$ \\
\hline M259T448 & Alpha-D-glucose 1-phosphate & 1.955748269 & 1.727049692 & $6.43531 \mathrm{E}-07$ \\
\hline М303T435 & N-acetylaspartylglutamate (NAAG) & 1.092531363 & 0.622574707 & 1.04037E-06 \\
\hline
\end{tabular}


Table 1 Metabolites that were altered significantly after GOLPH3L silencing (Continued)

\begin{tabular}{|c|c|c|c|c|}
\hline Name & ID & VIP & Fold change & $p$-value \\
\hline M145T372 & L-glutamine & 4.84006701 & 1.691397723 & $1.33369 \mathrm{E}-06$ \\
\hline M117T380_2 & Succinate & 3.22018177 & 0.745112292 & $1.33821 \mathrm{E}-06$ \\
\hline M146T390_2 & L-glutamate & 6.383368133 & 0.805813766 & $1.34212 \mathrm{E}-06$ \\
\hline M111T83 & Uracil & 4.093084571 & 0.338717051 & $1.98724 \mathrm{E}-06$ \\
\hline M255T38 & Palmitic acid & 15.91857778 & 1.482478443 & 2.63587E-06 \\
\hline M462T486 & Adenylsuccinic acid & 1.400896234 & 0.601445092 & $3.04252 \mathrm{E}-06$ \\
\hline M179T387_2 & Myo-Inositol & 1.869509045 & 0.876085103 & 3.53295E-06 \\
\hline M588T446 & GDP-L-fucose & 1.036473053 & 0.727980689 & 3.8859E-06 \\
\hline M458T140 & L-palmitoylcarnitine & 1.71058671 & 0.69922387 & 3.95794E-06 \\
\hline M296T70 & S-methyl-5'-thioadenosine & 2.695515248 & 0.562035793 & $5.0904 \mathrm{E}-06$ \\
\hline M114T308 & L-Proline & 1.492638552 & 0.844853133 & $6.29786 \mathrm{E}-06$ \\
\hline М130T386 & $\mathrm{N}$-acetyl-L-alanine & 1.284618775 & 0.714233544 & 7.07193E-06 \\
\hline M611T488 & Glutathione disulfide & 6.326051929 & 0.673543892 & 2.60415E-05 \\
\hline M89T221_2 & DL-lactate & 6.180555782 & 0.771109869 & $3.15076 \mathrm{E}-05$ \\
\hline M506T481 & Adenosine $5^{\prime}$-triphosphate (ATP) & 6.265508138 & 0.776594367 & 7.93354E-05 \\
\hline M133Т396 & L-malic acid & 3.710482706 & 0.870781902 & 0.00015885 \\
\hline M306Т394 & Glutathione & 6.846802373 & 0.645572726 & 0.000202429 \\
\hline M452T199 & 1-Palmitoyl-2-hydroxy-sn-glycero-3-phosphoethanolamine & 2.364541907 & 1.17565739 & 0.000288876 \\
\hline M281T38 & Oleic acid & 10.56865128 & 1.261282292 & 0.000386302 \\
\hline M337Т38 & Erucic acid & 1.462001645 & 0.804834608 & 0.000563427 \\
\hline M483T490 & Uridine 5'-triphosphate (UTP) & 2.985361942 & 0.791767071 & 0.000765923 \\
\hline M673Т189 & 1-Palmitoyl-2-oleoyl-sn-glycero-3-phosphate & 1.958401653 & 1.956770427 & 0.001001254 \\
\hline M253T38 & Cis-9-palmitoleic acid & 3.035713741 & 1.176091575 & 0.004203955 \\
\hline M227T150 & Myristic acid & 1.009572648 & 1.48607569 & 0.004811555 \\
\hline M197T38 & 3-Hydroxydodecanoic acid & 2.666768268 & 0.71831232 & 0.006944812 \\
\hline M333T125 & Penicillin G & 1.82556849 & 0.584544716 & 0.009905013 \\
\hline M273T34 & Salicylamide & 1.383050648 & 0.362734521 & 0.009978679 \\
\hline M579T469 & Uridine 5'-diphosphoglucuronic acid (UDP-D-glucuronate) & 3.315658544 & 0.86375998 & 0.010231094 \\
\hline M141T353 & 2-Oxoadipic acid & 11.40052894 & 0.912787301 & 0.015048419 \\
\hline М130T344 & Creatine & 1.009533641 & 0.795141329 & 0.017407461 \\
\hline M154T411 & Urocanic acid & 1.176119208 & 1.475399848 & 0.020677476 \\
\hline M173Т432 & Cis-Aconitate & 1.169850112 & 0.768659387 & 0.021442597 \\
\hline M219T2 & 4-Nonylphenol & 2.042585348 & 1.263228143 & 0.043438355 \\
\hline M134T153 & Adenine & 1.249418803 & 0.62240901 & 0.043527968 \\
\hline M269T136 & Heptadecanoic acid & 1.030910387 & 0.689149164 & 0.05747134 \\
\hline M131T375 & L-asparagine & 1.700684605 & 1.62285133 & 0.061339629 \\
\hline M191T497 & Citrate & 2.212863083 & 0.850809508 & 0.067218448 \\
\hline M181T296 & D-threitol & 1.061736173 & 0.563835355 & 0.079699611 \\
\hline
\end{tabular}

Legend: Untargeted metabolomics sequencing was used to reveal metabolites that were altered significantly after GOLPH3L silencing, and those with $p$-values $<$ 0.05 are indicated

reporter assay confirmed that miRNA-1185-2-3p regulated the levels of GOLPH3L mRNA by targeting the predicted sites within the $3^{\prime}$-UTR of GOLPH3L (Fig. 3a). Based on the GEO dataset GSE45666, we found that miRNA-1185-2-3p was expressed at a higher level in tumor-adjacent normal tissues than in breast tumor tissues and was inversely correlated with the prognosis of breast cancer patients (Fig. 3b, left panel). Consistently, the expression of miRNA-1185$2-3 p$ was higher in various breast cancer cell lines 
Table 2 KEGG pathway analysis of different metabolites that were altered significantly after GOLPH3L silencing

\begin{tabular}{|c|c|c|c|c|c|}
\hline Map-ID & Map name & Ref_per & $P$-value & FDR & Rich factor \\
\hline map 05230 & Central carbon metabolism in cancer & 0.902218971 & $1.52023 \mathrm{E}-15$ & $1.9611 \mathrm{E}-13$ & 0.378378378 \\
\hline map 00250 & Alanine, aspartate and glutamate metabolism & 0.682760302 & 1.32499E-09 & 8.54619E-08 & 0.321428571 \\
\hline map 02010 & $A B C$ transporters & 3.340648622 & 4.36608E-09 & 1.6293E-07 & 0.116788321 \\
\hline map 00010 & Glycolysis/Gluconeogenesis & 0.780297488 & 5.05208E-09 & $1.6293 \mathrm{E}-07$ & 0.28125 \\
\hline map 04974 & Protein digestion and absorption & 1.146061936 & 1.30029E-08 & 3.35475E-07 & 0.212765957 \\
\hline map 00970 & Aminoacyl-tRNA biosynthesis & 1.267983419 & 4.83446E-07 & $1.03941 \mathrm{E}-05$ & 0.173076923 \\
\hline map 01230 & Biosynthesis of amino acids & 3.121189954 & 7.70926E-07 & $1.42071 \mathrm{E}-05$ & 0.1015625 \\
\hline map 04978 & Mineral absorption & 0.707144599 & 9.11103E-07 & 1.46915E-05 & 0.24137931 \\
\hline map 00020 & Citrate cycle (TCA cycle) & 0.48768593 & 1.42224E-06 & 2.03854E-05 & 0.3 \\
\hline map 00230 & Purine metabolism & 2.316508169 & 1.61025E-06 & 2.07723E-05 & 0.115789474 \\
\hline map 00240 & Pyrimidine metabolism & 1.584979273 & 3.42162E-06 & 4.01262E-05 & 0.138461538 \\
\hline map 04080 & Neuroactive ligand-receptor interaction & 1.267983419 & 5.53193E-06 & 5.94683E-05 & 0.153846154 \\
\hline map 00480 & Glutathione metabolism & 0.926603267 & 6.39595E-06 & $6.34675 \mathrm{E}-05$ & 0.184210526 \\
\hline map 04922 & Glucagon signaling pathway & 0.633991709 & 7.69409E-06 & 7.08956E-05 & 0.230769231 \\
\hline map 04142 & Lysosome & 0.097537186 & $2.8207 \mathrm{E}-05$ & 0.00024258 & 0.75 \\
\hline map 00520 & Amino sugar and nucleotide sugar metabolism & 2.633504023 & 3.71809E-05 & 0.000299771 & 0.092592593 \\
\hline map 05231 & Choline metabolism in cancer & 0.268227262 & 3.99483E-05 & 0.000303137 & 0.363636364 \\
\hline map 00564 & Glycerophospholipid metabolism & 1.267983419 & 5.44277E-05 & 0.000390065 & 0.134615385 \\
\hline map 04068 & FoxO signaling pathway & 0.121921483 & $6.95244 \mathrm{E}-05$ & 0.000472034 & 0.6 \\
\hline map 01100 & Metabolic pathways & 65.88636918 & $9.23119 E-05$ & 0.000595411 & 0.025166543 \\
\hline map 00630 & Glyoxylate and dicarboxylate metabolism & 1.511826384 & 0.000171042 & 0.001050688 & 0.112903226 \\
\hline map 00190 & Oxidative phosphorylation & 0.390148744 & 0.000204512 & 0.001149292 & 0.25 \\
\hline map 04216 & Ferroptosis & 0.707144599 & 0.000204912 & 0.001149292 & 0.172413793 \\
\hline map 04924 & Renin secretion & 0.414533041 & 0.00026349 & 0.001359611 & 0.235294118 \\
\hline map 04964 & Proximal tubule bicarbonate reclamation & 0.414533041 & 0.00026349 & 0.001359611 & 0.235294118 \\
\hline map 04931 & Insulin resistance & 0.463301634 & 0.000416546 & 0.00206671 & 0.210526316 \\
\hline map 05012 & Parkinson disease & 0.48768593 & 0.000513007 & 0.002451034 & 0.2 \\
\hline map 04727 & GABAergic synapse & 0.219458669 & 0.000551859 & 0.002542494 & 0.333333333 \\
\hline map 04152 & AMPK signaling pathway & 0.536454523 & 0.000751888 & 0.00334244 & 0.181818182 \\
\hline map 04022 & cGMP-PKG signaling pathway & 0.243842965 & 0.000777312 & 0.00334244 & 0.3 \\
\hline map 04714 & Thermogenesis & 0.56083882 & 0.000896782 & 0.003731772 & 0.173913043 \\
\hline map 00052 & Galactose metabolism & 1.12167764 & 0.001825222 & 0.007357924 & 0.108695652 \\
\hline map 04150 & mTOR signaling pathway & 0.097537186 & 0.002198433 & 0.008593874 & 0.5 \\
\hline map 00620 & Pyruvate metabolism & 0.755913192 & 0.002830833 & 0.010740513 & 0.129032258 \\
\hline map 05131 & Shigellosis & 0.390148744 & 0.003333451 & 0.012286147 & 0.1875 \\
\hline map 01523 & Antifolate resistance & 0.414533041 & 0.003991299 & 0.014302156 & 0.176470588 \\
\hline map 01210 & 2-Oxocarboxylic acid metabolism & 3.267495733 & 0.004212296 & 0.014686113 & 0.059701493 \\
\hline map 00061 & Fatty acid biosynthesis & 1.414289198 & 0.00508542 & 0.017263662 & 0.086206897 \\
\hline map 04918 & Thyroid hormone synthesis & 0.512070227 & 0.007380826 & 0.024413503 & 0.142857143 \\
\hline map 04925 & Aldosterone synthesis and secretion & 0.536454523 & 0.008427501 & 0.02717869 & 0.136363636 \\
\hline map 00290 & Valine, leucine and isoleucine biosynthesis & 0.56083882 & 0.009557109 & 0.027345439 & 0.130434783 \\
\hline map 00220 & Arginine biosynthesis & 0.56083882 & 0.009557109 & 0.027345439 & 0.130434783 \\
\hline map 04724 & Glutamatergic synapse & 0.195074372 & 0.009751087 & 0.027345439 & 0.25 \\
\hline map 04211 & Longevity regulating pathway & 0.195074372 & 0.009751087 & 0.027345439 & 0.25 \\
\hline
\end{tabular}


Table 2 KEGG pathway analysis of different metabolites that were altered significantly after GOLPH3L silencing (Continued)

\begin{tabular}{|c|c|c|c|c|c|}
\hline Map-ID & Map name & Ref_per & $P$-value & FDR & Rich factor \\
\hline map 04740 & Olfactory transduction & 0.195074372 & 0.009751087 & 0.027345439 & 0.25 \\
\hline map 05032 & Morphine addiction & 0.195074372 & 0.009751087 & 0.027345439 & 0.25 \\
\hline map 04024 & CAMP signaling pathway & 0.609607413 & 0.012070106 & 0.033128589 & 0.12 \\
\hline map 05034 & Alcoholism & 0.243842965 & 0.015279969 & 0.041064916 & 0.2 \\
\hline map 00310 & Lysine degradation & 1.316752012 & 0.020299998 & 0.053442852 & 0.074074074 \\
\hline map 00760 & Nicotinate and nicotinamide metabolism & 1.341136308 & 0.021575257 & 0.054211054 & 0.072727273 \\
\hline map 04742 & Taste transduction & 0.755913192 & 0.021700254 & 0.054211054 & 0.096774194 \\
\hline map 04721 & Synaptic vesicle cycle & 0.292611558 & 0.021852518 & 0.054211054 & 0.166666667 \\
\hline map 01200 & Carbon metabolism & 2.779809802 & 0.022892225 & 0.055718812 & 0.052631579 \\
\hline map 00410 & Beta-alanine metabolism & 0.780297488 & 0.023615333 & 0.056414406 & 0.09375 \\
\hline map 00471 & D-Glutamine and D-glutamate metabolism & 0.316995855 & 0.025502823 & 0.059815712 & 0.153846154 \\
\hline map 00400 & Phenylalanine, tyrosine and tryptophan biosynthesis & 0.829066081 & 0.027712438 & 0.06383758 & 0.088235294 \\
\hline map 04611 & Platelet activation & 0.341380151 & 0.029381868 & 0.065349326 & 0.142857143 \\
\hline map 04923 & Regulation of lipolysis in adipocytes & 0.341380151 & 0.029381868 & 0.065349326 & 0.142857143 \\
\hline map 04066 & HIF-1 signaling pathway & 0.365764448 & 0.033479574 & 0.073201103 & 0.133333333 \\
\hline map 00500 & Starch and sucrose metabolism & 0.902218971 & 0.034522413 & 0.074223189 & 0.081081081 \\
\hline map 00561 & Glycerolipid metabolism & 0.926603267 & 0.036968063 & 0.078178362 & 0.078947368 \\
\hline map 00280 & Valine, leucine and isoleucine degradation & 1.024140454 & 0.047613224 & 0.097493744 & 0.071428571 \\
\hline map 00650 & Butanoate metabolism & 1.024140454 & 0.047613224 & 0.097493744 & 0.071428571 \\
\hline
\end{tabular}

Legend: KEGG pathways of different metabolites that were altered significantly after GOLPH3L silencing, $p$-values $<0.05$

than in the normal breast epithelial cell line MCF10A (Fig. 3b, right panel). To determine the impact of miRNA-1185-2-3p on the expression of GOLPH3L, we used miRNA mimics and inhibitors to demonstrate that the expression levels of miRNA-1185-2-3p were inversely correlated with the expression of GOLPH3L in breast cancer lines (Fig. $3 c$ and S2b). These results support the notion that miRNA-1185-2$3 p$ directly targets GOLPH3L mRNA.

Since miRNA-1185-2-3p suppresses the expression of GOLPH3L in breast cancer cells, we induced it in the breast cancer cell lines T47D and BT474 to determine whether miRNA-1185-2-3p could have the same tumor suppressive effects as GOLPH3L knockdown. The overexpression of miRNA-1185-2-3p also inhibited proliferation, survival, and migration and arrested the cell cycle of breast cancer cells (Fig. 3d-g and S2c-f). In addition, the induction of miRNA-1185-2-3p suppressed the tumorigenesis induced by GOLPH3L overexpression (Figure S3). Therefore, these data indicate that miRNA$1185-2-3 p$ could inhibit breast tumorigenesis by suppressing the expression of GOLPH3L.

\section{GOLPH3L affects glucose metabolism in breast Cancer cells}

To understand the mechanism by which GOLPH3L promotes tumorigenesis, we combined metabolomic analysis and transcriptome sequencing in GOLPH3Lsilenced T47D breast cancer cells. We identified metabolites that were altered significantly after GOLPH3L silencing. Notably, glycolytic intermediates such as acetyl coenzyme A (acetyl-CoA), adenosine monophosphate (AMP), adenosine $5^{\prime}$-diphosphate (ADP) and adenosine $5^{\prime}$-triphosphate (ATP) were reduced when GOLPH3L expression was downregulated (Fig. 4a, S4a and Table 1). KEGG pathway analysis showed that GOLPH3L expression could markedly influence central carbon metabolism in cancer, glycolysis/gluconeogenesis and 61 other pathways (Fig. 4b and Table 2). Correlation analysis of different metabolites revealed that acetyl-CoA, AMP, ADP and ATP were positively correlated in both positive ion mode (Fig. 4c, top panel) and negative ion mode (Fig. 4c, bottom panel). Since GOLPH3L knockdown decreased the proliferation of breast cancer cells, we evaluated the impact of GOLPH3L on metabolism and found that the suppression of GOLPH3L expression decreased glycolytic activity in breast cell lines (Fig. 4d and e).

Correspondingly, high-throughput transcriptome sequencing revealed that GOLPH3L expression could markedly alter the expression of 205 genes in breast cancer cells (Fig. 5a and Table 3), and the central carbon metabolism pathway was one of the 


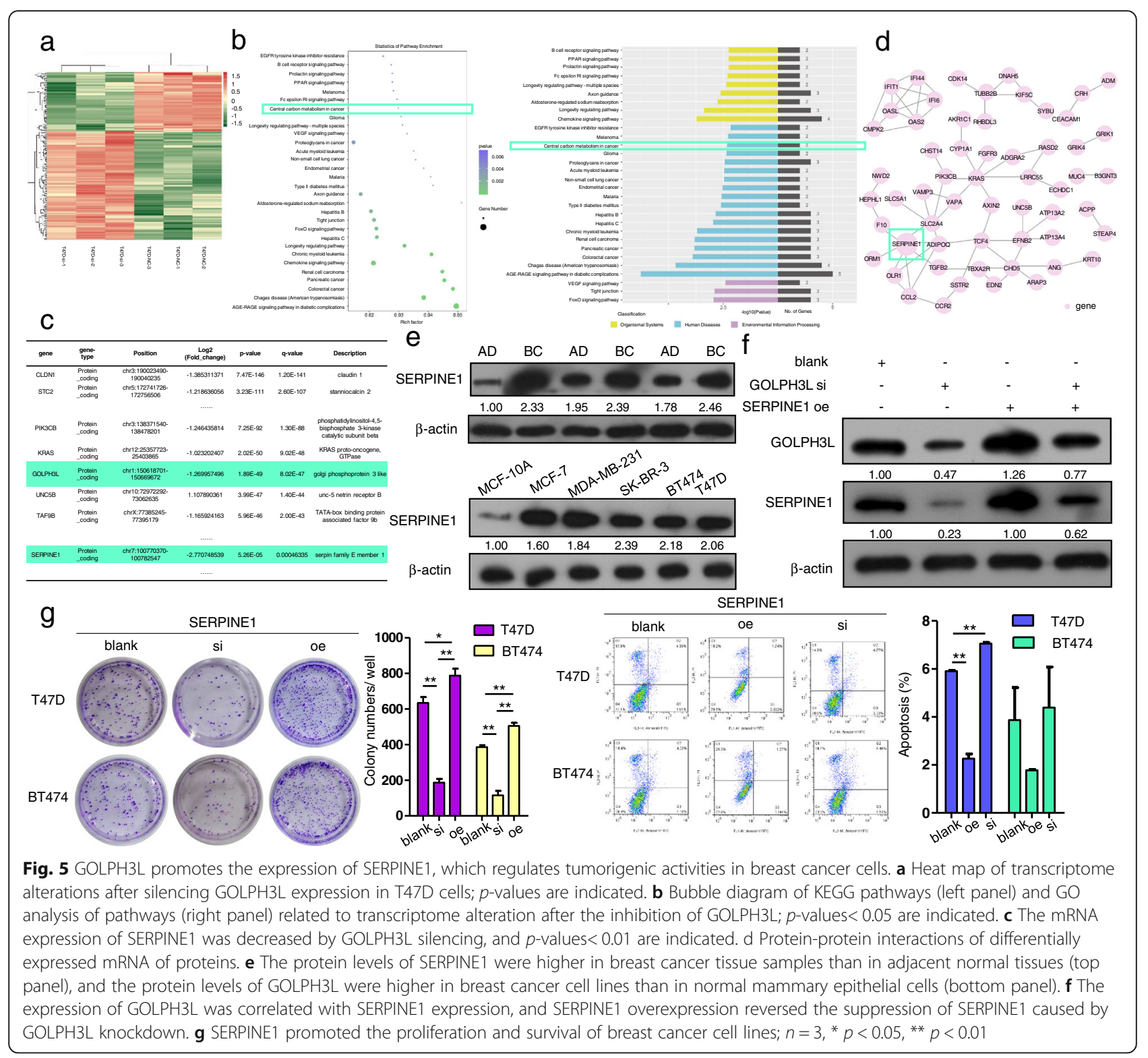

downregulated pathways in GOLPH3L-knockdown cancer cells (Fig. 5b and Table 4). Because GOLPH3L is located in the Golgi apparatus and may participate in mediating recruitment to Golgi membranes, we predicted that GOLPH3L likely functions by regulating the stability of other proteins involved in tumorigenesis via certain types of glycosylation. Therefore, we chose SERPINE1 as a candidate target through comprehensively analyzing the upregulated tumor suppressor genes and downregulated oncogenes that were related to the central carbon metabolism pathway and had glycosylation as a posttranslational modification (Fig. 5c and d).
GOLPH3L stabilizes p53-induced SERPINE1 expression in breast Cancer cells is positively correlated with increased glycolysis

Published data [28] and our KEGG analysis results (Figure $\mathrm{S4c}$ ) indicated that the p53 signaling pathway regulates SERPINE1 and therefore suppresses tumor cell proliferation, invasion and migration, whereas it promotes cell apoptosis. To assess the role of SERPINE1 in breast cancer, we examined the expression level of SERP INE1. When compared to those in the tumor-adjacent normal tissues and normal breast epithelial cell line MCF-10A, the expression levels of SERPINE1 were increased in breast cancer tissues and breast cancer cell 
Table 3 A total of 205 differentially expressed genes were found in T47D cells after GOLPH3L knockdown

\begin{tabular}{|c|c|c|}
\hline $\begin{array}{l}\text { Compared } \\
\text { group }\end{array}$ & Upregulated genes & Downregulated genes \\
\hline \multirow[t]{2}{*}{ NC--si } & 133 & 72 \\
\hline & $\begin{array}{l}\text { CP, KRT10, PAPSS2, CASP7, UNC5B, AKR1C2, SPRY4, CHST14, FGFR3, } \\
\text { SLC6A14, LRRC55, ILF3-AS1, EXOC3-AS1, APLNR, CDK14, AKR1C1, } \\
\text { IFI6, OAS2, AXIN2, ZNF702P, MAN1B1-AS1, IFIT1, C2CD4A, AKR1C3, } \\
\text { MPV17 L, HABP4, ZCCHC24, PCP4 L1, RASD2, TUB, CRH, STMN3, } \\
\text { PALMD, GRIK4, CHD5, FL37453, HOXB7, SLC16A10, STEAP4, ARAP3, } \\
\text { LOC100132249, SSTR2, SLC5A1, EGR3, ENSG00000261040.2, OASL, } \\
\text { ADAM19, TDO2, GAREM2, ENSG00000261071.1, CEACAM1, THEM5, } \\
\text { WFDC21P, ADGRA2, ZNF98, PPP1R1B, CDSN, IFI44, SMCO4, } \\
\text { SLC22A31, PCDH20, GDF1, COLCA1, C1 1orf220, B3GNT3, ORM1, } \\
\text { ENSG00000254024.1, ADORA1, IFI27, CMPK2, GLTPD2, CCDC177, } \\
\text { MYEOV, ENSG00000269962.1, PTCHD1, ENSG00000266795.2, STRA6, } \\
\text { RHBDL3, FLJ6000, ANG, RAMP2-AS1, PYDC1, TBXA2R, NELL2, } \\
\text { LOC100996419, DLG5-AS1, SLC2A4, GAPDHP43, MYLK-AS1, VGF, } \\
\text { EDN2, AMPD3, CNTFR, LOXL4, INSM1, ENSG00000251417.1, KCNB1, } \\
\text { ST7-AS1, HMGB1P1, EIF4HP1, GPRC5B, ETV1, ENSG00000233029.3, } \\
\text { SNX25P1, ENSG00000273284.1, LINC01023,4-Sep, OGFRP1, TUBB2B, } \\
\text { C1S, ENSG00000270964.1, F11-AS1, ENSG00000256982.1, C11 orf45, } \\
\text { ENSG00000261693.1, F10, ENSG00000236514.1, ZNF663P, TOLLIP- } \\
\text { AS1, ALOX12B, TNFAIP8 L3, UNC5CL, VANGL2, ENSG00000269289.1, } \\
\text { FRMPD3, PPP1R14BP3, LINC00865, USH1G, ACOT11, HEPHL1, } \\
\text { ATP13A4, MUC4, NKX1-2 }\end{array}$ & $\begin{array}{l}\text { CLDN1, STC2, FAM65C, CRIM1, PIK3CB, CNOT6, VAPA, REEP3, PON2, } \\
\text { AVL9, TMEM45B, VAMP3, KRAS, GOLPH3L, TAF9B, ATP13A2, OLR1, } \\
\text { EFNB2, LMBRD1, MAP 6D1, TGFB2, ANXA3, PHACTR1, SHISA2, } \\
\text { SH3RF1, USP2, POLR3G, ANKFN1, FSIP2, SAP30 L-AS1, TGFB2-OT1, } \\
\text { GRIK1, ACPP, SYNPO, BCHE, ABCC13, PDZK1, DNAH5, SCART1, } \\
\text { CCL2, SYBU, ECHDC1, ENSG00000260037.1, LOC100288911, } \\
\text { ENSG00000265242.1, SERPINE1, ADIPOQ, ENSG00000254343.2, } \\
\text { NWD2, CCR2, KIF5C, OTOR, TCF4, GPR183, SNORD12B, LINC00312, } \\
\text { MUM1 L1, TAB3-AS1, CYP1A1, ENSG00000267896.1, PSG9, SPOCK2, } \\
\text { ENSG00000269680.1, SLCO2A1, ADM, ENSG00000255142.1, PLAC4, } \\
\text { ENSG00000257193.1, ENSG00000231868.1, CRYGS, IL16, } \\
\text { ENSG00000236933.1 }\end{array}$ \\
\hline
\end{tabular}

Legends: KEGG pathways of different metabolites that were altered significantly after GOLPH3L silencing, $p$-values $<0.05$

lines (Fig. 5e). In addition, the protein levels of SERP INE1 were correlated with the protein levels of GOLPH3L in breast cancer cells, supporting the notion that GOLPH3L may regulate the expression of SERP INE1 (Fig. 5f). Moreover, the expression of SERPINE1 promoted breast tumorigenesis (Fig. 5g). Using a coimmunoprecipitation (Co-IP) assay, we confirmed the interaction between GOLPH3L and SERPINE1 in breast cancer cells (Fig. 6a). To test whether GOLPH3L can stabilize SERPINE1, the alteration of the expression of GOLPH3L significantly affected the half-life of SERP INE1 in a breast cancer cell line, indicating that GOLPH3L contributes to the stabilization of SERPINE1 (Fig. 6b). Subsequently, we altered the expression of GOLPH3L in T47D cells, and the IP results showed increased ubiquitination of SERPINE1 in the presence of GOLPH3L (Fig. 6c). Furthermore, the overexpression of SERPINE1 reversed the antitumor activities induced by the suppression of GOLPH3L in breast cancer cell lines (Fig. 6d and e). Therefore, GOLPH3L promotes glucose metabolism in breast cancer and is conducive to SERP INE1 stabilization.

\section{Discussion}

Breast cancer cells are produced by mutations in the DNA of normal breast cells, genetics and other factors, such as diet and exercise. Lifestyle habits can also be the cause of DNA mutations. Some DNA mutations are heritable, but the vast majority of DNA changes are acquired, and such acquired mutations occur only in breast cancer cells [29]. Mutated DNA causes corresponding changes in genes that regulate cell proliferation and apoptosis, which can lead to uncontrolled proliferation and apoptosis, thus promoting the development of tumors. According to the New York Cancer Institute [30], targeted therapeutics can be categorized according to their targets: those that target the regulatory mechanism of tumor formation, those that target the tumor microenvironment, those that target the tumor immune system, those that target tumor markers and those that target tumor stem cells. Dysregulation of targets that regulate tumor formation can stimulate a series of downstream signaling pathways, resulting in abnormal division, growth, metabolism, cell environment and angiogenesis of tumor cells compared with normal cells.

Golgi phosphoprotein 3-like (GOLPH3L) is a prognostic biomarker of cervical cancer [24] and ovarian cancer [25] and may be a mitochondrial biogenesis marker in breast cancer metabolism [31]. However, the roles of GOLPH3L in breast tumorigenesis remain unclear. SERPINE1 promotes breast cancer cell metastasis [32] and glycolytic metabolism in triple-negative breast cancer (TNBC) [33] and participates in EGFR signaling [34]. We discovered that GOLPH3L promotes glucose metabolism in breast cancer cells by stabilizing SERPINE1, which is regulated by p53 transcription [35]. In this context, we demonstrate that silencing the GOLPH3L gene suppresses breast tumorigenesis and glucose metabolism, while overexpression of GOLPH3L promotes breast tumorigenesis. Therefore, GOLPH3L-mediated stabilization of SERPINE1 could represent an important oncogenic pathway in the glucose metabolism of breast cancer. Our findings suggest that 
Table 4 KEGG pathways involved in GOLPH3L-silenced breast cancer cells

\begin{tabular}{|c|c|c|c|c|}
\hline Database & ID & $P$-Value & Corrected $P$-Value & Genes \\
\hline KEGG PATHWAY & hsa04933 & $5.42713 \mathrm{E}-07$ & $7.32662 \mathrm{E}-05$ & CCL2;PIK3CB;SERPINE1;KRAS;TGFB2 \\
\hline KEGG PATHWAY & hsa05142 & 2.07617E-05 & 0.001401414 & CCL2;PIK3CB;TGFB2;SERPINE1 \\
\hline KEGG PATHWAY & hsa05210 & 0.000126423 & 0.003878901 & PIK3CB;TGFB2;KRAS \\
\hline KEGG PATHWAY & hsa05212 & 0.000151007 & 0.003878901 & PIK3CB;TGFB2;KRAS \\
\hline KEGG PATHWAY & hsa05211 & 0.000157602 & 0.003878901 & PIK3CB;TGFB2;KRAS \\
\hline KEGG PATHWAY & hsa04062 & 0.00018996 & 0.003878901 & CCL2;PIK3CB;KRAS;CCR2 \\
\hline KEGG PATHWAY & hsa05220 & 0.000201128 & 0.003878901 & PIK3CB;TGFB2;KRAS \\
\hline KEGG PATHWAY & hsa04211 & 0.000412618 & 0.006962933 & PIK3CB;KRAS;ADIPOQ \\
\hline KEGG PATHWAY & hsa05160 & 0.001101508 & 0.01518735 & PIK3CB;KRAS;CLDN1 \\
\hline KEGG PATHWAY & hsa04068 & 0.001124989 & 0.01518735 & PIK3CB;TGFB2;KRAS \\
\hline KEGG PATHWAY & hsa04530 & 0.001247139 & 0.015305797 & VAPA;KRAS;CLDN1 \\
\hline KEGG PATHWAY & hsa05161 & 0.001431726 & 0.016106915 & PIK3CB;TGFB2;KRAS \\
\hline KEGG PATHWAY & hsa04960 & 0.001705229 & 0.017708152 & PIK3CB;KRAS \\
\hline KEGG PATHWAY & hsa04360 & 0.002413822 & 0.022159731 & EFNB2;PIK3CB;KRAS \\
\hline KEGG PATHWAY & hsa04930 & 0.002525744 & 0.022159731 & PIK3CB;ADIPOQ \\
\hline KEGG PATHWAY & hsa05144 & 0.002626339 & 0.022159731 & CCL2;TGFB2 \\
\hline KEGG PATHWAY & hsa05213 & 0.00293929 & 0.023341421 & PIK3CB;KRAS \\
\hline KEGG PATHWAY & hsa05223 & 0.003382408 & 0.024852551 & PIK3CB;KRAS \\
\hline KEGG PATHWAY & hsa05221 & 0.003497766 & 0.024852551 & PIK3CB;KRAS \\
\hline KEGG PATHWAY & hsa05205 & 0.003682986 & 0.024860155 & PIK3CB;TGFB2;KRAS \\
\hline KEGG PATHWAY & hsa04370 & 0.003977354 & 0.025568702 & PIK3CB;KRAS \\
\hline KEGG PATHWAY & hsa04213 & 0.004355952 & 0.026010475 & PIK3CB;KRAS \\
\hline KEGG PATHWAY & hsa05214 & 0.004485724 & 0.026010475 & PIK3CB;KRAS \\
\hline KEGG PATHWAY & hsa05230 & 0.004750595 & 0.026010475 & PIK3CB;SERPINE1 \\
\hline KEGG PATHWAY & hsa04664 & 0.004885683 & 0.026010475 & PIK3CB;KRAS \\
\hline KEGG PATHWAY & hsa05218 & 0.005301493 & 0.026010475 & PIK3CB;KRAS \\
\hline KEGG PATHWAY & hsa04917 & 0.005443594 & 0.026010475 & PIK3CB;KRAS \\
\hline KEGG PATHWAY & hsa03320 & 0.005443594 & 0.026010475 & OLR1;ADIPOQ \\
\hline KEGG PATHWAY & hsa04662 & 0.005587435 & 0.026010475 & PIK3CB;KRAS \\
\hline KEGG PATHWAY & hsa01521 & 0.006800173 & 0.030390563 & PIK3CB;KRAS \\
\hline KEGG PATHWAY & hsa05166 & 0.006978574 & 0.030390563 & PIK3CB;TGFB2;KRAS \\
\hline KEGG PATHWAY & hsa04060 & 0.007424504 & 0.031322125 & CCL2;TGFB2;CCR2 \\
\hline KEGG PATHWAY & hsa04012 & 0.007950284 & 0.032246156 & PIK3CB;KRAS \\
\hline KEGG PATHWAY & hsa05215 & 0.008121254 & 0.032246156 & PIK3CB;KRAS \\
\hline KEGG PATHWAY & hsa05323 & 0.008468143 & 0.032662838 & CCL2;TGFB2 \\
\hline KEGG PATHWAY & hsa01522 & 0.009548044 & 0.034763406 & PIK3CB;KRAS \\
\hline KEGG PATHWAY & hsa04914 & 0.009733696 & 0.034763406 & PIK3CB;KRAS \\
\hline KEGG PATHWAY & hsa04915 & 0.009920954 & 0.034763406 & PIK3CB;KRAS \\
\hline KEGG PATHWAY & hsa05146 & 0.010109813 & 0.034763406 & PIK3CB;TGFB2 \\
\hline KEGG PATHWAY & hsa05231 & 0.010300268 & 0.034763406 & PIK3CB;KRAS \\
\hline KEGG PATHWAY & hsa04066 & 0.010685948 & 0.035185438 & PIK3CB;SERPINE1 \\
\hline KEGG PATHWAY & hsa04660 & 0.011077952 & 0.035607703 & PIK3CB;KRAS \\
\hline KEGG PATHWAY & hsa04668 & 0.012085376 & 0.037712623 & CCL2;PIK3CB \\
\hline KEGG PATHWAY & hsa04725 & 0.012291521 & 0.037712623 & PIK3CB;KRAS \\
\hline
\end{tabular}


Table 4 KEGG pathways involved in GOLPH3L-silenced breast cancer cells (Continued)

\begin{tabular}{|c|c|c|c|c|}
\hline Database & ID & $P$-Value & Corrected $P$-Value & Genes \\
\hline KEGG PATHWAY & hsa04919 & 0.013777442 & 0.039775364 & PIK3CB;KRAS \\
\hline KEGG PATHWAY & hsa04670 & 0.013777442 & 0.039775364 & PIK3CB;CLDN1 \\
\hline KEGG PATHWAY & hsa05145 & 0.013995787 & 0.039775364 & PIK3CB;TGFB2 \\
\hline KEGG PATHWAY & hsa04722 & 0.014215636 & 0.039775364 & PIK3CB;KRAS \\
\hline KEGG PATHWAY & hsa04071 & 0.014436984 & 0.039775364 & PIK3CB;KRAS \\
\hline KEGG PATHWAY & hsa04152 & 0.015337266 & 0.041410618 & PIK3CB;ADIPOQ \\
\hline KEGG PATHWAY & hsa04380 & 0.016969364 & 0.044918905 & PIK3CB;TGFB2 \\
\hline KEGG PATHWAY & hsa04650 & 0.01769056 & 0.045927416 & PIK3CB;KRAS \\
\hline KEGG PATHWAY & hsa04910 & 0.018672129 & 0.047302645 & PIK3CB;KRAS \\
\hline KEGG PATHWAY & hsa04210 & 0.018921058 & 0.047302645 & PIK3CB;KRAS \\
\hline KEGG PATHWAY & hsa04550 & 0.019423126 & 0.047674946 & PIK3CB;KRAS \\
\hline KEGG PATHWAY & hsa04072 & 0.019930779 & 0.048047414 & PIK3CB;KRAS \\
\hline KEGG PATHWAY & hsa05200 & 0.021609077 & 0.05050568 & PIK3CB;TGFB2;KRAS \\
\hline KEGG PATHWAY & hsa04932 & 0.021751022 & 0.05050568 & PIK3CB;ADIPOQ \\
\hline KEGG PATHWAY & hsa04390 & 0.022551551 & 0.05050568 & TGFB2;SERPINE1 \\
\hline KEGG PATHWAY & hsa04150 & 0.022551551 & 0.05050568 & PIK3CB;KRAS \\
\hline KEGG PATHWAY & hsa04145 & 0.022821085 & 0.05050568 & VAMP3;OLR1 \\
\hline KEGG PATHWAY & hsa04921 & 0.023637696 & 0.051469176 & PIK3CB;KRAS \\
\hline KEGG PATHWAY & hsa05164 & 0.028783631 & 0.06167921 & CCL2;PIK3CB \\
\hline KEGG PATHWAY & hsa05168 & 0.031818708 & 0.067117587 & CCL2;TAF9B \\
\hline KEGG PATHWAY & hsa04977 & 0.036402182 & 0.075604532 & LMBRD1 \\
\hline KEGG PATHWAY & hsa05169 & 0.037582935 & 0.076394178 & POLR3G;PIK3CB \\
\hline KEGG PATHWAY & hsa05203 & 0.037914147 & 0.076394178 & PIK3CB;KRAS \\
\hline KEGG PATHWAY & hsa04015 & 0.039924992 & 0.079262851 & PIK3CB;KRAS \\
\hline KEGG PATHWAY & hsa04810 & 0.041287714 & 0.08078031 & PIK3CB;KRAS \\
\hline KEGG PATHWAY & hsa04320 & 0.042104357 & 0.081201261 & KRAS \\
\hline KEGG PATHWAY & hsa05216 & 0.04352471 & 0.082758252 & KRAS \\
\hline KEGG PATHWAY & hsa04014 & 0.045835719 & 0.085941973 & PIK3CB;KRAS \\
\hline KEGG PATHWAY & hsa00640 & 0.047773358 & 0.0871541 & ECHDC1 \\
\hline KEGG PATHWAY & hsa03020 & 0.047773358 & 0.0871541 & POLR3G \\
\hline
\end{tabular}

Legends: KEGG pathways of different metabolites that were altered significantly after GOLPH3L silencing, $p$-values $<0.05$

GOLPH3L functions as an oncogene in breast cancer by promoting cellular proliferation and migration and suppressing apoptosis. Considering the tumorigenic role of GOLPH3L, we also investigated the upstream regulators of GOLPH3L. Fortunately, we identified that miR-1185-2-3p directly targets the sites within the 3'-UTR of GOLPH3L and functionally suppresses the expression of GOLPH3L. In support of this notion, we demonstrated that miR-1185-2-3p inhibits breast tumorigenesis by suppressing GOLPH3L expression. This is the first time that miR-1185-2-3p has been identified as a regulator in cancer development. Therefore, it will be important to identify the pathway related to miR-1185-2-3p.
The treatment and prognosis of breast cancer differs according to the expression of different molecular makers such as estrogen receptor (ER), progesterone receptor (PR) and human epidermal growth factor 2 (HER2). HER2+ breast cancers tend to grow and spread more aggressively. Different types of drugs such as monoclonal antibodies and kinase inhibitors have been developed to target the HER2 protein [36, 37]. Approximately 2 of 3 breast cancers are hormone receptor-positive $(\mathrm{ER}+$ or $\mathrm{PR}+)$. Treatment with hormone therapy is often helpful in these cases, and certain targeted therapy drugs, such as CDK4/6 inhibitors [13], mTOR inhibitor (Everolimus) and PI3K inhibitor (Alpelisib) [38], can increase the 


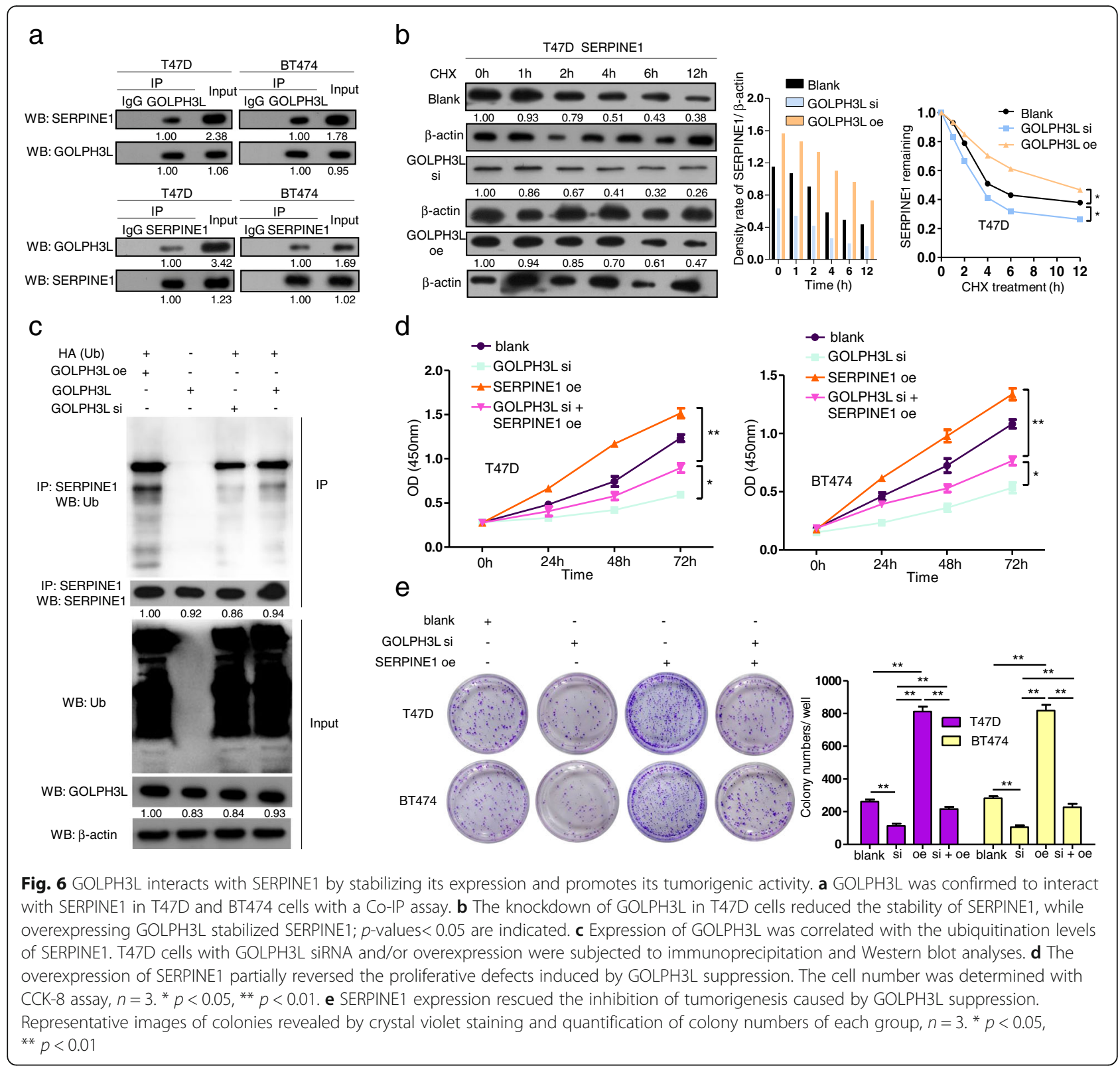

efficacy of hormone therapy. Among all types of breast cancer, triple-negative breast cancer (TNBC) has the worst prognosis due to the lack of an effective target. Moreover, over $30 \%$ of breast cancer patients may suffer recurrence. In terms of metastatic breast cancer, systemic therapy usually shows unsatisfactory treatment effects. Therefore, it is of great clinical significance to discover new therapeutic targets for breast cancer. Oncogenic proteins such as hypoxia-inducible factor, Myc, p53 and PI3K/Akt/ mTOR pathway proteins, which are involved in metabolic reprogramming [39], may serve as candidate anticancer agents. Several preclinical trials have shown effectiveness of an inhibitor targeting the glycolytic pathway [40]. Our discovery provides a mechanistic link between miR-1185-2-3p, GOLPH3L and SERP INE1; this pathway plays a significant role in glucose metabolism in breast cancer and may serve as a novel therapeutic target for breast cancer.

\section{Conclusions}

We discovered a functional pathway linking miR1185-2-3p, GOLPH3L and SERPINE1 that plays a significant role in glucose metabolism in breast cancer and provides new therapeutic targets for breast cancer treatment. 


\section{Supplementary Information}

The online version contains supplementary material available at https://doi. org/10.1186/s13046-020-01767-9.

Additional file 1:Supplementary Figure 1. GOLPH3L regulated the tumorigenic activities of BT474 cells. (A) Knockdown of GOLPH3L in MCF10A, BT474 and T47D cells with five distinct siRNAs. (B) The overexpression (oe) of GOLPH3L in MCF-10A, BT474 and T47D cells. (C) The expression of GOLPH3L in BT474 cells promotes their proliferation, $n=3,{ }^{*} p<$ $0.05,{ }^{* *} p<0.01$. (D) GOLPH3L expression suppresses the apoptosis of BT474 cells, $n=3$, ** $p<0.01$. (E) Knockdown of GOLPH3L inhibits the migration of BT474 cells using a transwell assay, $n=3,{ }^{*} p<0.05,{ }^{* *} p<0.01$. (F) The suppression of GOLPH3L significantly blocks the BT474 cell cycle at G0/ G1 phase, $n=3,{ }^{*} p<0.05$, ${ }^{* *} p<0.01$. Supplementary Figure 2 . miR-1185-2-3p negatively regulates the tumorigenesis of BT474 cells. (A) The predicted miRNAs which were most likely to regulate with GOLPH3L. (B) The overexpression of miR-1185-2-3p was achieved with miRNA mimics and inhibition miR-1185-2-3p was achieved with miRNA inhibitor in T47D and BT474, $n=3,{ }^{* *} p<0.01$. (C) The overexpression of miR-1185$2-3 p$ inversely correlated with the proliferation of BT474 cells, $n=3$, ** $p<0.01$. (D) The overexpression of miR-1185-2-3p promoted the apoptosis of BT474 cells, $n=3,{ }^{*} p<0.05$. (E) miR-1185-2-3p overexpression could significantly inhibited the migration of BT474 cells, $n=3$, ${ }^{*} p<0.05$. (F) The overexpression of miR-1185-2-3p could block the cell cycle at G0/ G1 phase, $n=3,{ }^{*} p<0.05$. Supplementary Figure 3. miR-1185-2-3p overexpression partially reversed the tumorigenesis induced by GOLPH3L overexpression. (A) The overexpression of miR-1185-2-3p inversely correlated with the up-regulation of proliferation induced by GOLPH3L overexpression in T47D cells, $n=3,{ }^{*} p<0.05$, ** $p<0.01$. (B) miR-1185-2-3p partially reversed the apoptosis induced by GOLPH3L overexpression in T47D cells, $n=3$. * $p<0.05$. (C) Overexpressed miR-1185-2-3p inhibited the up-regulation of migration caused by GOLPH3L up-regulation using transwell assay, $n=3 .{ }^{*} p<0.05$. (D) The overexpression of miR-1185-2-3p affected the cell cycle regulation by GOLPH3L, $n=3,{ }^{*} p<0.05$, ${ }^{* *} p<$ 0.01.Supplementary Figure 4. p53 pathway promotes the transcription of SERPINE1. (A) Volcano plots of fold changes and $p$-values of altered metabolites after GOLPH3L suppression in the Positive Ion Mode (top panel) and Negative Ion Mode (bottom panel). (B) Volcano plots of fold changes and $p$-values of RNA-seq data after silencing GOLPH3L expression in T47D cells. (C) STRING analysis predicted protein-protein network indicating that SERPINE1 interacted with p53 (top panel) and the role of SERPINE1 in p53 signaling pathway after GOLPH3L knockdown in T47D cells (bottom panel).

\section{Abbreviations}

GOLPH3L: Golgi phosphoprotein 3-like; SERPINE1: Serpin family E member 1; EGFR: Epidermal growth factor receptor; EdU: 5-ethynyl-2'- deoxyuridine

\section{Acknowledgements}

Not applicable.

\section{Authors' contributions}

YX performed the proliferation analysis of different cell lines, transcriptome sequencing analysis, and Western blot analysis and was a major contributor in writing the manuscript. WC analyzed the tumor metabolism sequencing data and carried out mechanistic research regarding Co-IP and seahorse assays. $J$ analyzed and interpreted the prognosis of human breast cancer patients and carried out an in vitro tumorigenesis experiment. XZ was responsible for preprocessing the clinical tissue regarding breast cancer and adjacent normal tissue. KJ performed all bioinformatic analyses in this study. JZ conducted all qPCR analyses in this study. SL established the transfected cell lines in this study. JW performed the transcriptome sequencing analysis regarding the miRNA candidate of the target gene. KX conducted the Transwell assay of different cell lines. ZH performed the apoptosis analysis of different cell lines. YY performed the cell cycle analysis of different cell lines. QL carried out the protein stabilization analysis in this study. PZ constructed the plasmids and cultured the cell lines in this study. YL took part in the statistical analysis of the dual-luciferase reporter assay and cell function in this research. LL took part in partial statistical analysis of Western blotting, GPCR and seahorse assays in this research. ML led the graduate students engaged in this study and interpreted all clinical data regarding clinical significance. WC was responsible for funding acquisition and led the researchers with a bachelor's degree engaged in this study. WH was responsible for funding acquisition and confirmed that data/figure presentation accurately reflected the original and oversaw the postdoctoral students who participated in this study. All authors read and approved the final manuscript.

\section{Funding}

This study is supported by the National Natural Science Foundation of China (Grant No. 81902865 and Grant No. 61427807), the China Postdoctoral Science Foundation (2019 M652974), Sanming Project of Medicine in Shenzhen (SZSM201612019), the Science and Technology Foundation of Jiangmen (JK [2020] No. 58-2020YLI010), the Medical Science and Technology Research Foundation of Guangdong Province (YWJKH [2020] No. 15A2020510), and the President's Fund of Nanfang Hospital, Southern Medical University (Grant No. 2019C017).

\section{Availability of data and materials}

The datasets used and/or analyzed during the current study are available from the corresponding author on reasonable request.

\section{Ethics approval and consent to participate}

All institutional and national guidelines for the care and use of laboratory animals were followed.

\section{Consent for publication}

Not applicable.

\section{Competing interests}

The authors declare that they have no competing interests.

\section{Author details}

${ }^{1}$ Taishan People's Hospital, Postdoctoral Innovation Practice Base of Southern Medical University, Taishan 529200, China. ${ }^{2}$ National Key Discipline of Human Anatomy, School of Basic Medical Science, Southern Medical University, Guangzhou 510515, China. ${ }^{3}$ Affiliated Hospital of Guangdong Medical University, Guangdong Medical University, Zhanjiang 524001, China. ${ }^{4}$ School of Basic Medical Science, Guangzhou University of Chinese Medicine, Guangzhou 510006, China. ${ }^{5}$ Breast Center, Department of general surgery, Nanfang Hospital, Southern Medical University, Guangzhou 510515, China. ${ }^{6}$ The Eighth Affiliated Hospital, Sun Yat-sen University, Shenzhen 518000, China. ${ }^{7}$ Department of Radiology, Nanfang Hospital, Southern Medical University, Guangzhou 510515, China. ${ }^{8}$ Department of Pathology, School of Basic Medicine, Guangdong Medical University, Zhanjiang 524023, China.

Received: 17 September 2020 Accepted: 5 November 2020

Published online: 28 January 2021

References

1. Bray F, Ferlay J, Soerjomataram I, Siegel RL, Torre LA, Jemal A. Global cancer statistics 2018: GLOBOCAN estimates of incidence and mortality worldwide for 36 cancers in 185 countries. CA Cancer J Clin. 2018;68:394-424.

2. Ren R, Sun H, Ma C, Liu J, Wang H. Colon cancer cells secrete exosomes to promote self-proliferation by shortening mitosis duration and activation of STAT3 in a hypoxic environment. Cell Biosci. 2019;9:62.

3. Janzen DM, Tiourin E, Salehi JA, Paik DY, Lu J, Pellegrini M, et al. Retraction note: an apoptosis-enhancing drug overcomes platinum resistance in a tumour-initiating subpopulation of ovarian cancer. Nat Commun. 2020;11: 2218.

4. Nyquist MD, Corella A, Coleman I, De Sarkar N, Kaipainen A, Ha G, et al. Combined TP53 and RB1 loss promotes prostate cancer resistance to a spectrum of therapeutics and confers vulnerability to replication stress. Cell Rep. 2020;31:107669.

5. Overdijk MB, Verploegen S, van den Brakel JH, van Bueren JJL, Vink T, van de Winkel JG, et al. Epidermal growth factor receptor (EGFR) antibodyinduced antibody-dependent cellular cytotoxicity plays a prominent role in inhibiting tumorigenesis, even of tumor cells insensitive to EGFR signaling inhibition. J Immunol. 2011;187:3383-90.

6. Niu Y, Bao L, Chen Y, Wang C, Luo M, Zhang B, et al. HIF2-induced long noncoding RNA RAB11B-AS1 promotes hypoxia-mediated angiogenesis and breast cancer metastasis. Cancer Res. 2020;80:964-75. 
7. Shaker H, Bundred NJ, Landberg G, Pritchard SA, Albadry H, Nicholson SL, et al. Breast cancer stromal clotting activation (tissue factor and thrombin): a pre-invasive phenomena that is prognostic in invasion. Cancer Med. 2020;9: 1768-78.

8. Zimmer AS, Van Swearingen AED, Anders CK. HER2-positive breast cancer brain metastasis: a new and exciting landscape. Cancer Rep (Hoboken). 2020:e1274. https://doi.org/10.1002/cnr2.1274.

9. Cantile M, Di Bonito M, Cerrone M, Collina F, De Laurentiis M, Botti G. Long non-coding RNA HOTAIR in breast cancer therapy. Cancers (Basel). 2020;12: 1197.

10. Di LJ, Byun JS, Wong MM, Wakano C, Taylor T, Bilke S, et al. Genome-wide profiles of CtBP link metabolism with genome stability and epithelial reprogramming in breast cancer. Nat Commun. 2013;4:1449.

11. Salas LA, Johnson KC, Koestler DC, O'Sullivan DE, Christensen BC. Integrative epigenetic and genetic pan-cancer somatic alteration portraits. Epigenetics. 2017;12:561-74.

12. Tahtouh R, Wardi L, Sarkis R, Hachem R, Raad I, El Zein N, et al. Glucose restriction reverses the Warburg effect and modulates PKM2 and mTOR expression in breast cancer cell lines. Cell Mol Biol (Noisy-le-grand). 2019;65: 26-33.

13. Cao J, Liu X, Yang Y, Wei B, Li Q, Mao G, et al. Decylubiquinone suppresses breast cancer growth and metastasis by inhibiting angiogenesis via the ROS/p53/ BAl1 signaling pathway. Angiogenesis. 2020;23:325-38.

14. Dennis JW, Nabi IR, Demetriou M. Metabolism, cell surface organization, and disease. Cell. 2009;139:1229-41.

15. Li CW, Lim SO, Chung EM, Kim YS, Park AH, Yao J, et al. Eradication of triplenegative breast cancer cells by targeting glycosylated PD-L1. Cancer Cell. 2018:33:187-201.e10

16. Vlodavsky I, Friedmann Y. Molecular properties and involvement of heparanase in cancer metastasis and angiogenesis. J Clin Invest. 2001;108: $341-7$.

17. Taniguchi N, Kizuka Y. Glycans and cancer: role of N-glycans in cancer biomarker, progression and metastasis, and therapeutics. Adv Cancer Res. 2015;126:11-51.

18. Caldwell SA, Jackson SR, Shahriari KS, Lynch TP, Sethi G, Walker S, et al. Nutrient sensor O-GICNAc transferase regulates breast cancer tumorigenesis through targeting of the oncogenic transcription factor FoxM1. Oncogene. 2010:29:2831-42.

19. Ma Z, Vosseller K. Cancer metabolism and elevated O-GICNAc in oncogenic signaling. J Biol Chem. 2014;289:34457-65.

20. Zhu W, Leber B, Andrews DW. Cytoplasmic O-glycosylation prevents cell surface transport of E-cadherin during apoptosis. EMBO J. 2001;20:59996007.

21. Yang WH, Kim JE, Nam HW, Ju JW, Kim HS, Kim YS, et al. Modification of p53 with O-linked $\mathrm{N}$-acetylglucosamine regulates p53 activity and stability. Nat Cell Biol. 2006;8:1074-83.

22. Tanaka H, Imamura N, Oguma N, Shintani T, Tanaka K, Hyodo H, et al. Acute myelogenous leukemia with PIG-A gene mutation evolved from aplastic anemia-paroxysmal nocturnal hemoglobinuria syndrome. Int J Hematol. 2001;73:206-12.

23. Kunigou O, Nagao H, Kawabata N, Ishidou Y, Nagano S, Maeda S, et al. Role of GOLPH3 and GOLPH3L in the proliferation of human rhabdomyosarcoma. Oncol Rep. 2011:26:1337-42.

24. Feng $Y$, He F, Yan S, Huang H, Huang Q, Deng T, et al. The role of GOLPH3L in the prognosis and NACT response in cervical cancer. J Cancer. 2017:8: 443-54

25. He S, Niu G, Shang J, Deng Y, Wan Z, Zhang C, et al. The oncogenic Golgi phosphoprotein 3 like overexpression is associated with cisplatin resistance in ovarian carcinoma and activating the NF-kappaB signaling pathway. J Exp Clin Cancer Res. 2017;36:137.

26. Kim J, Yu L, Chen W, Xu Y, Wu M, Todorova D, et al. Wild-type p53 promotes cancer metabolic switch by inducing PUMA-dependent suppression of oxidative phosphorylation. Cancer Cell. 2019;35:191-203.e8.

27. Kim J, Xu S, Xiong L, Yu L, Fu X, Xu Y. SALL4 promotes glycolysis and chromatin remodeling via modulating HP1alpha-Glut1 pathway. Oncogene. 2017;36:6472-9.

28. Wu DM, Wang S, Wen X, Han XR, Wang YJ, Fan SH, et al. MircoRNA-1275 promotes proliferation, invasion and migration of glioma cells via SERPINE1. J Cell Mol Med. 2018;22:4963-74.

29. Martinez-Ramos D, Simon-Monterde L, Queralt-Martin R, SuelvesPiqueres C, Menor-Duran P, Escrig-Sos J. Breast cancer in octogenarian. Are we doing our best? A population-registry based study. Breast. 2018;38:81-5.

30. Tang J, Pearce L, O'Donnell-Tormey J, Hubbard-Lucey VM. Trends in the global immuno-oncology landscape. Nat Rev Drug Discov. 2018; 17:922.

31. Sotgia F, Whitaker-Menezes D, Martinez-Outschoorn UE, Salem AF, Tsirigos A, Lamb R, et al. Mitochondria "fuel" breast cancer metabolism: fifteen markers of mitochondrial biogenesis label epithelial cancer cells, but are excluded from adjacent stromal cells. Cell Cycle. 2012;11:4390-401.

32. Zhang W, Xu J, Fang H, Tang L, Chen W, Sun Q, et al. Endothelial cells promote triple-negative breast cancer cell metastasis via PAl-1 and CCL5 signaling. FASEB J. 2018;32:276-88.

33. Humphries BA, Buschhaus JM, Chen YC, Haley HR, Qyli T, Chiang B, et al. Plasminogen activator inhibitor 1 (PAl1) promotes actin cytoskeleton reorganization and glycolytic metabolism in triple-negative breast cancer. Mol Cancer Res. 2019;17:1142-54.

34. Azimi I, Petersen RM, Thompson EW, Roberts-Thomson SJ, Monteith GR. Hypoxia-induced reactive oxygen species mediate $\mathrm{N}$-cadherin and SERPINE1 expression, EGFR signalling and motility in MDA-MB-468 breast cancer cells. Sci Rep. 2017;7:15140.

35. Szoltysek K, Janus P, Zajac G, Stokowy T, Walaszczyk A, Widlak W, et al. RRAD, IL4I1, CDKN1A, and SERPINE1 genes are potentially co-regulated by NF-kappaB and p53 transcription factors in cells exposed to high doses of ionizing radiation. BMC Genomics. 2018;19:813.

36. Chakraborty AK, Liang K, DiGiovanna MP. Co-targeting insulin-like growth factor I receptor and HER2: dramatic effects of HER2 inhibitors on nonoverexpressing breast cancer. Cancer Res. 2008;68: 1538.

37. Lategahn J, Hardick J, Grabe T, Niggenaber J, Jeyakumar K, Keul M, et al. Targeting Her2-insYVMA with covalent inhibitors-a focused compound screening and structure-based design approach. J Med Chem. 2020;63: 11725-55.

38. Kennedy SP, O'Neill M, Cunningham D, Morris PG, Toomey S, BlancoAparicio C, et al. Preclinical evaluation of a novel triple-acting PIM/ PI3K/mTOR inhibitor, IBL-302, in breast cancer. Oncogene. 2020;39: 3028-40.

39. Wang S, Wang N, Zheng Y, Yang B, Liu P, Zhang F, et al. Caveolin-1 inhibits breast cancer stem cells via c-Myc-mediated metabolic reprogramming. Cell Death Dis. 2020;11:450

40. Gandhi N, Das GM. Metabolic reprogramming in breast cancer and its therapeutic implications. Cells. 2019;8:89.

\section{Publisher's Note}

Springer Nature remains neutral with regard to jurisdictional claims in published maps and institutional affiliations.

Ready to submit your research? Choose BMC and benefit from:

- fast, convenient online submission

- thorough peer review by experienced researchers in your field

- rapid publication on acceptance

- support for research data, including large and complex data types

- gold Open Access which fosters wider collaboration and increased citations

- maximum visibility for your research: over $100 \mathrm{M}$ website views per year

At $\mathrm{BMC}$, research is always in progress.

Learn more biomedcentral.com/submissions 